\title{
On $E_{c}$-Open and $\delta$ - $\beta_{c}$-Open sets in Topological Spaces
}

\author{
Sarah Haqi Abdulwahid, Alaa. M. F. AL. Jumaili*
}

Department of Mathematics, College of Education for pure science, University of Anbar, Iraq

DOI: $10.36347 /$ sipms.2020.v07i08.005

| Received: 14.07.2020 | Accepted: 22.07.2020 | Published: 28.08.2020

*Corresponding author: Alaa. M. F. AL. Jumaili

Abstract

The main aim of the present this paper is to introduce and investigate some new classes of generalized open sets called $E_{c}$-open and $\delta$ - $\beta_{\mathrm{c}}$-open sets. The class of $E_{c}$-open sets lies strictly between the classes of $\delta$ - $\beta_{\mathrm{c}}$-open and $P_{c}$-open sets , as well the class of $\delta$ - $\beta_{\mathrm{c}}$-open sets is contained in the class of $\delta$ - $\beta$-open sets and properly contains all the classes of $\beta_{\mathrm{c}}-$ open, $b_{c}$-open, $S_{c}$-open and $E_{c}$-open sets. Several characterizations and fundamental properties concerning of these new forms of generalized open sets are obtained. Furthermore, the relationships among $E_{c}$-open and $\delta$ - $\beta_{c}$-open sets and other well-known types of generalized open sets are also investigated.

Keywords: $E_{c}$-open sets, $\delta$ - $\beta_{\mathrm{c}}$-open sets, $\delta$ - $\beta$-open sets, $E$-open sets.

Copyright @ 2020: This is an open-access article distributed under the terms of the Creative Commons Attribution license which permits unrestricted use, distribution, and reproduction in any medium for non-commercial use (NonCommercial, or CC-BY-NC) provided the original author and source are credited.

\section{INTRODUCTION}

It is known that generalized open sets play a very important role in general Topology and Indeed a significant theme in General Topology and Real analysis concerns the various modified forms of continuity, separation axioms etc. In recent years a number of generalizations of open sets have been considered such as: Semi-open sets, $\alpha$-open sets, preopen sets, semi-pre-open sets, $b$-open sets, $b$ - $\theta$-open sets, $\beta$ - $\theta$-op en sets, $\delta$-pre-open sets, $\delta$ - $\beta$-open sets, $E$ open sets, $E$ - $\theta$-open and $\delta-\beta-\theta$-open sets play an important role in generalization of continuity in topological spaces.

A new classes of generalized open sets in a topological space, called $\delta$ - $\beta$-open sets or $\mathrm{e}^{*}$-open sets was introduced and some of its properties were obtained by E. Hatir and T. Noiri [1] and Erdal Ekici [2], in [3] Hariwan Z. Ibrahim presented a new class of $b$-open sets called $\mathrm{B}_{\mathrm{c}}$-open, this class of sets lies strictly between the classes of $\theta$-semi open and b-open sets, moreover, Alias B. Khalaf, Zanyar A. Ameen, in [4] introduced a new class of sets, called $\mathrm{S}_{\mathrm{c}}$-open sets, and investigate some properties of $\mathrm{S}_{\mathrm{c}}$-continuity and in [5] Zanyar A. Ameen, introduced a new class of sets, called $\mathrm{P}_{\mathrm{c}}$-open sets, and investigate some properties of $\mathrm{P}_{\mathrm{c}^{-}}$ continuity. As well, Ayman Y. Mizyed, in [6] studied a new class of generalized open sets called $\beta_{\mathrm{c}}$-Open Sets which is contained in the class of $\beta$-open sets and contains the class of $\mathrm{B}_{\mathrm{c}}$-open sets and he introduced $\beta_{\mathrm{c}^{-}}$ continuous functions as a new class of generalized continuous functions and gave some characterizations of these functions.

In recent years, many researchers studied deferent forms of continuous mappings utilize new generalized of open sets. E. Hatir and T. Noiri [1] and Erdal Ekici [2] introduced $\delta$ - $\beta$-continuous and $\mathrm{e}^{*}$ continuous mappings respectively, and they obtained some new decompositions of continuity, J. H. Park [7] introduced a new class of functions called strongly $\theta-b$ continuous functions which is a generalization of both strongly $\theta$-pre-continuous functions and strongly $\theta$ semi-continuous functions, some characterizations and several properties concerning strongly $\theta-b$-continuous functions are obtained, as well in [8] Alaa. M. F, and Xiao-Song Yang, defined a new class of mappings called strong continuous functions called strongly $\theta-\delta$ $\beta$-continuous by using two new strong forms of $\delta-\beta$ open sets.

The purpose of the present paper is to introduce and study new classes of generalized open sets called $E_{c}$-open and $\delta$ - $\beta_{\mathrm{c}}$-open sets. Several characterizations and basic properties concerning of these kinds of generalized open sets are obtained, the relationships among these kinds of generalized open sets and other well-known types of generalized open sets are also discussed. In addition several topological properties concerning of $E_{c}$ (resp. $\delta$ - $\beta_{\mathrm{c}}$ )-Neighborhood, $E_{c}\left(\right.$ resp. $\delta$ - $\beta_{\mathrm{c}}$ )-Interior, $E_{c}$ (resp. $\delta$ - $\beta_{\mathrm{c}}$ )-Closure, $E_{c}$ (resp. $\delta$ - $\left.\beta_{\mathrm{c}}\right)$-Derived and $E_{c}$ (resp. $\delta$ - $\left.\beta_{\mathrm{c}}\right)$-Frontier of a sets are introduced. 


\section{PRELIMINARIES}

Throughout this paper, $(X, T)$ and $\left(Y, T^{*}\right)$ (or simply $X$ and $Y$ ) mean topological spaces on which no separation axioms are assumed unless explicitly stated. For any subset $\mathrm{A}$ of $X$, The closure and interior of $\mathrm{A}$ are denoted by $C l(A)$ and $\operatorname{Int}(A)$, respectively. We recall the following required definitions and results of generalized open sets, which will be used often throughout this paper.

Definition 2.1: Let $(X, T)$ be a topological space. A subset $A$ of $X$ is said to be:

a) Regular open (resp. regular closed) [9] if $A=$ $\operatorname{Int}(\mathrm{Cl}(A))($ resp. $A=\mathrm{Cl}(\operatorname{Int}(A)))$.

b) $\delta$-open [10] if for each $\varkappa \in A$ there exists a regular open set $V$ such that $\varkappa \in V \subseteq A$. The $\delta$ interior of $A$ is the union of all regular open sets contained in $A$ and is denoted by $\operatorname{Int}_{\delta}(A)$. The subset $A$ is called $\delta$-open [10] if $A=\operatorname{Int}_{\delta}(A)$. A point $\varkappa \in X$ is called a $\delta$-cluster points of $A$ [10] if $A \cap \operatorname{Int}(\mathrm{Cl}(V)) \neq \varphi$, for each open set $V$ containing $\varkappa$. The set of all $\delta$-cluster points of $A$ is called the $\delta$ closure of $A$ and is denoted by $C l_{\delta}(A)$. If $A=$ $\mathrm{Cl}_{\delta}(A)$ ), then $\mathrm{A}$ is said to be $\delta$-closed [10]. The complement of $\delta$-closed set is said to be $\delta$-open set. A subset $A$ of a Topological space $X$ is called $\delta$ open [10] if for each $\varkappa \in A$ there exists an open set $G$ such that, $\varkappa \in G \subseteq \operatorname{Int}(\mathrm{Cl}(G)) \subseteq A$. The family of all $\delta$-open sets in $X$ is denoted by $\delta \Sigma(X, T)$.

c) $\alpha$-open [11] (resp. semi-open [12], pre-open [13], $\beta$-open [14] or semi-pre-open [15], b-open [16] or $\gamma$-open [17], $\delta$-pre-open [18]) if $\mathrm{A} \subseteq$ $\operatorname{Int}(\mathrm{Cl}(\operatorname{Int}(\mathrm{A}))) \quad($ resp. $\quad A \subseteq \mathrm{Cl}(\operatorname{Int}(\mathrm{A})), \quad \mathrm{A} \subseteq$ $\operatorname{Int}(\mathrm{Cl}(\mathrm{A})), \quad \mathrm{A} \subseteq \mathrm{Cl}(\operatorname{Int}(\mathrm{Cl}(\mathrm{A}))), \quad \mathrm{A} \subseteq \operatorname{Int}(\mathrm{Cl}(\mathrm{A})) \mathrm{U}$ $\mathrm{Cl}(\operatorname{Int}(\mathrm{A})), \mathrm{A} \subseteq \operatorname{Int}\left(\mathrm{Cl}_{\delta}(\mathrm{A})\right)$.

Remark 2.2: The complement of a semi-open (resp. $\alpha$ open, pre-open, $\beta$-open, b-open, $\delta$-pre-open) set is said to be semi-closed [19], (resp. $\alpha$-closed [20], pre-closed [21], $\beta$-closed [13], b-closed [17], $\delta$-pre-closed [18]. The intersection of all b-closed (resp. semi-closed, $\alpha-$ closed, pre-closed, $\beta$-closed, $\delta$-pre-closed) sets of $X$ containing $A$ is called the $b$-closure [17] (resp. s-closure [19], $\alpha$-closure [11], pre-closure [21], $\beta$-closure [14], $\delta$ pre-closure [18]) of $\mathrm{A}$ and are denoted by $b \mathrm{Cl}(A)$, (resp. $\mathrm{SCl}(A), \alpha \mathrm{Cl}(A), P C l(A), \beta \mathrm{Cl}(A), \delta P C l(A))$.

Remark 2.3: The family of all $b$-open (resp. $\beta$-open, $\alpha$ open, semi-open, pre-open, $\delta$-pre-open and regular open) subsets of $X$ containing a point $\varkappa \in X$ is denoted by $B \Sigma(X, \varkappa)$ (resp. $\beta \Sigma(X, \varkappa), \alpha \Sigma(X, \varkappa), S \Sigma(X, \varkappa), P \Sigma(X$, $\varkappa), \delta P \Sigma(X, \varkappa)$ and $R \Sigma(X, \varkappa)$ ), The family of all b-open (resp. $\beta$-open, $\alpha$-open, semi-open, pre-open, $\delta$-pre-open and regular open) sets in $X$ are denoted by $B \Sigma(X, T)$ (resp. $\beta \Sigma(X, T), \alpha \Sigma(X, T), S \Sigma(X, T), P \Sigma(X, T), \delta P \Sigma(X, T)$ and $R \Sigma(X, T)$ ).

Definition 2.4: Let $(X, T)$ be a Topological space. Then: a) A subset $A$ of a space $X$ is called $E$-open [22] if $A \subseteq \mathrm{Cl}(\delta$-Int $(A)) \cup \operatorname{Int}(\delta-\mathrm{Cl}(A))$. The complement of an $E$-open set is called $E$-closed. The intersection of all $E$-closed sets containing $A$ is called the $E$-closure of $A$ [22] and is denoted by $E$ $\mathrm{Cl}(A)$. The union of all $E$-open sets of $X$ contained in $A$ is called the $E$-interior [22] of $A$ and is denoted by $E$-Int $(A)$.

b) A subset $\mathrm{A}$ of a space $X$ is called $\delta$ - $\beta$-open [1] or $\mathrm{e}^{*}$-open [2], if $A \subseteq \mathrm{Cl}(\operatorname{Int}(\delta-\mathrm{Cl}(A)))$, the complement of a $\delta$ - $\beta$-open set is called $\delta$ - $\beta$-closed. The intersection of all $\delta$ - $\beta$-closed sets containing $A$ is called the $\delta$ - $\beta$-closure of $A$ [1] and is denoted by $\delta-\beta-\mathrm{Cl}(A)$. The union of all $\delta$ - $\beta$-open sets of $X$ contained in $A$ is called the $\delta$ - $\beta$-interior [1] of $A$ and is denoted by $\delta-\beta-\operatorname{Int}(A)$.

Remark 2.5: The family of all $E$-open (resp. $E$-closed, $\delta$ - $\beta$-open, $\delta$ - $\beta$-closed) subsets of $X$ containing a point $\varkappa$ $\in X$ is denoted by $E \Sigma(X, \varkappa)$ (resp. $E C(X, \varkappa), \delta-\beta \Sigma(X, \varkappa)$, $\delta$ - $\beta C(X, \varkappa))$. The family of all $E$-open (resp. $E$-closed, $\delta$ - $\beta$-open, $\delta$ - $\beta$-closed) sets in $X$ is denoted by $E \Sigma(X, T)$ (resp. $E C(X, T), \delta-\beta \Sigma(X, T), \delta-\beta C(X, T)$ ).

Definition 2.6: Let $(X, T)$ be a Topological space, then:

a) A subset $A$ of $X$ is said to be $\theta$-open [10] if for each $\varkappa \in A \exists$ an open set $G$ such that, $\varkappa \in G \subseteq$ $\mathrm{Cl}(G) \subseteq A$. (i. e) A point $\varkappa \in X$ is called a $\theta$ cluster point of $A$ if $\operatorname{Cl}(V) \cap A \neq \varphi$ for every open subset $\mathrm{V}$ of $X$ containing $\varkappa$. The set of all $\theta$-cluster points of $\mathrm{A}$ is called the $\theta$-closure of $A$ and is denoted by $\mathrm{Cl}_{\theta}(A)$. If $A=\mathrm{Cl}_{\theta}(A)$, then $A$ is said to be $\theta$-closed [10]. The complement of a $\theta$-closed set is said to be $\theta$-open. The family of all $\theta$-open sets in $X$ is denoted by $\theta \Sigma(X, T)$.

b) A subset $A$ of $X$ is said to be $\theta$-Semi-open [23] if for each $\varkappa \in A$ there exists a Semi-open set $G$ such that, $\varkappa \in G \subseteq C l(G) \subseteq A$. The family of all $\theta$ Semi-open sets in $X$ is denoted by $\operatorname{\theta S} \Sigma(X, T)$.

Remark 2.7: The collection of $\theta$-open sets in a Topological space $X$ forms a Topology $T_{\theta}$ which is coarser than $T$. as well, the family of $\delta$-open sets in a Topological space $X$ forms a Topology $T_{\delta}$ such that $T_{\delta}$ $\subseteq T$.

Proposition 2.8: [24] A Topological space $(X, T)$ is Regular if and only if $T_{\theta}=T$.

Definitions 2.9: A Topological space $(X, T)$ is said to be:

a) An extremally disconnected (resp. A locally indiscrete) [25] if the closure of every open set of $X$ is open in $X$ (resp. if and only if every open set is closed).

b) A regular space [26] if for each $\varkappa \in X$ and for each open set $G$ containing $\varkappa$, there exist an open set $K$ such that, $x \in K \subseteq \mathrm{Cl}(K) \subseteq G$.

c) Alexandroff space [27] if any arbitrary intersection of open sets is open. 
Remark 2.10: A space $X$ is Alexandroff-Space $\Leftrightarrow$ arbitrary union of closed sets is closed.

Proposition 2.11: [22, 28] the following properties hold for a space $X$ :

a) The Arbitrary union of any family of $E$-(resp. $\delta$ $\beta$ )-open sets in $X$, is an $E$ - $($ resp. $\delta$ - $\beta$ )-open set. b) The Arbitrary intersection of any family of $E$ (resp. $\delta$ - $\beta$ )-closed sets in $X$, is an $E$ - $($ resp. $\delta-\beta)$ closed set.

Remark 2.12: We have the following diagram in which the converses of implications need not be true, see the examples in $[28,22,2]$.

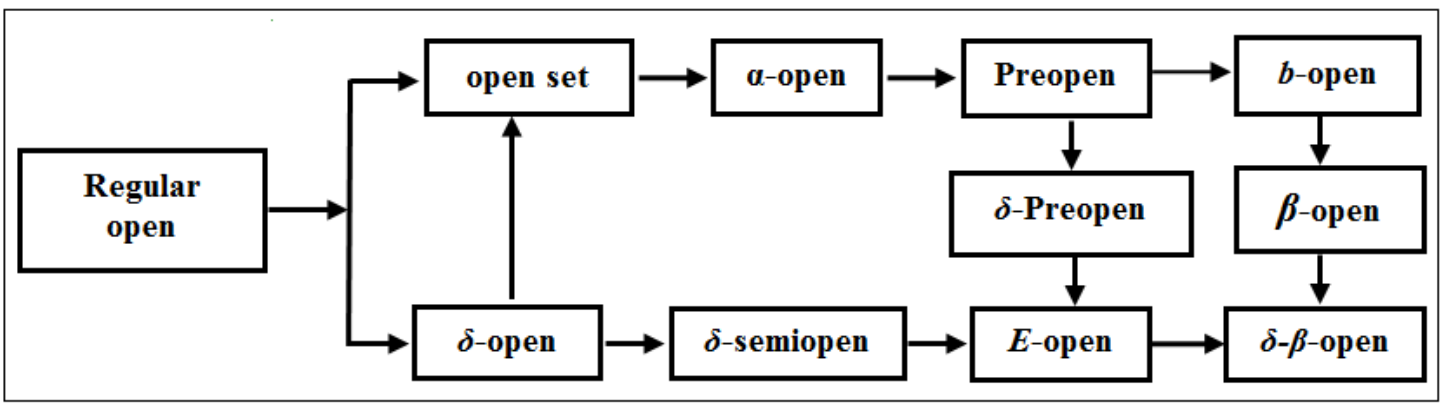

Fig-1: The relationships among some well-known generalized open sets in Topological Spaces

Lemma 2.13: [29] Let $X$ be a space and $A, B \subseteq X$. if $A \in \quad \delta \Sigma(X) \quad$ and $B \in \boldsymbol{E} \Sigma(X)($ resp. $B \in \boldsymbol{\delta}-\beta \Sigma(X))$ then, $A \cap B \in \boldsymbol{E} \Sigma(X)($ resp. $A \cap B \in \boldsymbol{\delta}-\beta \Sigma(X))$.

Lemma 2.14: $[30,18]$ If $A \subseteq X^{*} \subseteq X \& X^{*} \in E \Sigma(X, T)$ (resp. $X^{*} \in \delta-\beta \Sigma(X, T)$ ). Then $A \in E \Sigma(X, T)$ (resp. $A \in$ $\delta-\beta \Sigma(X, T)) \Leftrightarrow A \in E \Sigma\left(X^{*}, T\right)\left(\right.$ resp. $\left.A \in \delta-\beta \Sigma\left(X^{*}, T\right)\right)$.

Lemma 2.15: $[28,22]$ If $U \in E \Sigma(X)($ resp. $\delta$ - $\beta \Sigma(X)) \&$ $V \in E \Sigma(Y)($ resp. $\delta$ - $\beta \Sigma(Y))$, then $U \times V \in E \Sigma(X \times Y)$ (resp. $\boldsymbol{\delta}-\boldsymbol{\beta} \Sigma(X \times Y))$.

Theorem 2.16: Let $\left(Y, T_{Y}\right)$ be a subspace of a space $(X$, $T$ ). If $A$ is a closed subset in $Y$ and $\mathrm{Y} \subseteq \mathrm{X}$, then $A$ is closed in $X$ [31].

\section{CHARACTERIZATIONS OF $\left(E_{c}\right)$ AND $\left(\delta-\beta_{c}\right)$-OPEN SETS}

In this section, we consider a new classes of generalized open sets called $E_{\mathrm{c}}$-open and $\delta$ - $\beta_{c}$-open sets and several characterizations concerning of these forms of generalized open sets are obtained. Furthermore, the relations among $E_{c-}$ (resp. $\delta$ - $\beta_{\mathrm{c}}$ )-open sets and other forms of generalized open sets are discussed.

Definition 3.1: Let $(X, T)$ be a Topological space. A subset $A$ of $X$ is said to be:

a) $\quad B_{\mathrm{c}}$-open [3] if $\forall \varkappa \in A \in B O(X, T), \exists$ a closed set $F$ such that, $\varkappa \in F \subseteq A$.

b) $S_{\mathrm{c}}$-open [4] if $\forall \varkappa \in A \in S O(X, T), \exists$ a closed set $F$ such that, $x \in F \subseteq A$.

c) $\quad P_{\mathrm{c}}$-open [5] if $\forall \varkappa \in A \in P O(X, T), \exists$ a closed set $F$ such that, $\varkappa \in F \subseteq A$.

d) $\beta_{\mathrm{c}}$-open [6] if $\forall \varkappa \in A \in \beta O(X, T), \exists$ a closed set $F$ such that, $\varkappa \in F \subseteq A$.

e) The family of all $B_{\mathrm{c}}$-open (resp. $S_{\mathrm{c}}$-open, $P_{\mathrm{c}^{-}}$ open, $\beta_{\mathrm{c}}$-open) sets in $X$ are denoted by $B C \Sigma(X)($ resp. $S C \Sigma(X), P C \Sigma(X), \beta C \Sigma(X))$.
Definition 3.2: Let $(X, T)$ be a Topological space. A subset $A$ of $X$ is said to be:

I. $\quad E_{\mathrm{c}}$-open set if for each $\varkappa \in A \in E \Sigma(X, T)$, there exists a closed set $F$ such that, $\varkappa \in F \subseteq A$. The family of all $E_{\mathrm{c}}$-open subsets of $(X, T)$ is denoted by $E C \Sigma(X, T)$ OR $E C \Sigma(X)$.

II. $\delta$ - $\beta_{\mathrm{c}}$-open set if for each $\varkappa \in A \in \delta-\beta \Sigma(X, T)$, there exists a closed set $F$ such that, $\varkappa \in F \subseteq A$. The family of all $\delta-\beta_{\mathrm{c}}$-open subsets of $(X, T)$ is denoted by $\delta-\beta C \Sigma(X, T)$ OR $\delta-\beta C \Sigma(X)$.

III. A subset $F$ of a space $(X, T)$ is said to be $E_{c}$ (resp. $\delta$ - $\beta_{\mathrm{c}}$ )-closed set when, $X \backslash F \in E C \Sigma(X, T)$ (resp. $\delta-\beta C \Sigma(X, T)$ ).

Remark 3.3: The family of all $E_{c}\left(\right.$ resp. $\delta$ - $\left.\beta_{\mathrm{c}}\right)$-closed subsets of $(X, T)$ is denoted by $\operatorname{ECC}(X, T)$ OR $\operatorname{ECC}(X)$ (resp. $\delta$ - $\beta C C(X, T)$ OR $\delta$ - $\beta C C(X)$ ).

Theorem 3.4: Let $(X, T)$ be a Topological space. A subset $A$ of space $X$ is $E_{c}$ (resp. $\delta$ - $\beta_{\mathrm{c}}$ )-open set iff $A$ is $E$ (resp. $\delta$ - $\beta$ )-open set and it is a union of closed sets. That is $A=\bigcup F_{\lambda}$ where $A$ is $E_{c}$ (resp. $\delta$ - $\beta_{\mathrm{c}}$ )-open set and $F_{\lambda}$ closed sets $\forall \lambda$.

Proof: Let $(X, T)$ be a Topological space and $A$ be a $E_{c}$ (resp. $\delta$ - $\beta_{\mathrm{c}}$ )-open set. Then, $A$ is $E$ - $($ resp. $\delta$ - $\beta$ )-open and $\forall \lambda \in A \exists a$ closed $F_{\lambda}$ in $X$ (s. $\left.t\right) \quad \lambda \in F_{\lambda} \subseteq A \Rightarrow$ $\bigcup_{\lambda \in A} F_{\lambda} \subseteq A \subseteq \bigcup_{\lambda \in A} F_{\lambda}$. Hence, $A=\bigcup_{\lambda \in A} F_{\lambda}$ where $F_{\lambda}$ closed sets $\forall \lambda \in A$. The converse follows directly from the definitions of $E_{c}$ (resp. $\delta$ - $\beta_{\mathrm{c}}$ )-open sets.

Remark 3.5: From the respective definitions, the relationships among $E_{c}$ (resp. $\delta$ - $\beta_{\mathrm{c}}$ )-open sets and other well-known forms of generalized open sets are shown in the following figure: 


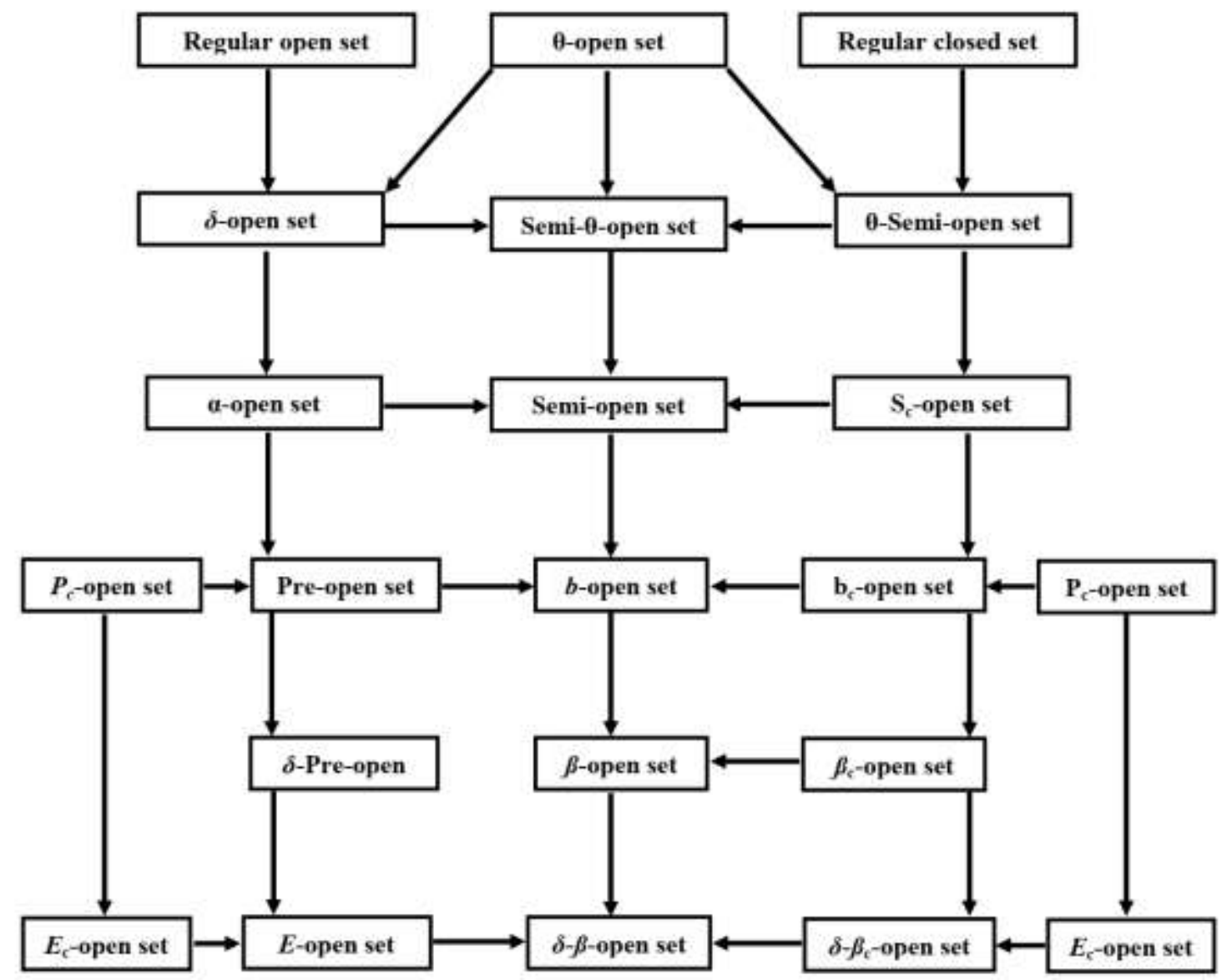

Fig-2: The relationships among $E_{c}\left(\right.$ resp. $\delta$ - $\beta_{c}$ )-open sets and other well-known types of generalized open sets

However none of these implications is reversible as shown via examples of $[7,8,9,10,11,27]$ and the following examples:

Example 3.6: Let $X=\{\varkappa, y, w, z\}$ and let $T=\{\varphi,\{\varkappa\}$, $\{w\},\{\varkappa, y\},\{\varkappa, w\},\{\varkappa, y, w\},\{\varkappa, w, z\}, X\}$. Then the family of all closed subsets is: $T^{c}=\{X,\{y, w, z\},\{\varkappa, y$, $z\},\{w, z\},\{y, z\},\{z\},\{y\}, \varphi\}$. Thus:

i. The set $\{y, w\}$ is $E$-open but it is not $E_{c}$-open set.

ii. The set $\{y, w, z\}$ is $E_{c}$-open set but it is not $S_{\mathrm{c}^{-}}$ open and not $P_{\mathrm{c}}$-open, also it is neither $b_{\mathrm{c}}$-open nor $\beta_{\mathrm{c}}$-open set.

iii. The set $\{y, z\}$ is $\delta$ - $\beta_{\mathrm{c}}$-open set but it is not $E_{c^{-}}$ open and not $S_{\mathrm{c}}$-open. Also it is not $P_{\mathrm{c}}$-open and neither $b_{\mathrm{c}}$-open nor $\beta_{\mathrm{c}}$-open set.

iv. Let $X=\{\varkappa, y, w, z, s\}$ and let $T=\{\varphi,\{\varkappa, y\}$, $\{w, z\},\{\varkappa, y, w, z\}, X\}$. Then the family of all closed subsets is: $T^{c}=\{X,\{w, z, s\},\{\varkappa, y, s\}$, $\{s\}, \varphi\}$.Then, the subset $\{\varkappa, s\}$ is $\delta$ - $\beta$-open but it is not $\delta$ - $\beta_{\mathrm{c}}$-open set.

Remark 3.7: The following example explains that a $E_{c}$ (resp. $\delta-\beta_{\mathrm{c}}$ )-open sets not necessary to be closed set.

Examples 3.8: Let $\left(R, T_{u}\right)$ be the usual Topological space, and let $A$ be set of all rational numbers, obvious that $A$ is $E($ resp. $\delta$ - $\beta)$-open set and since $\forall \varkappa \in A, \varkappa \in$ $\{\varkappa\} \subseteq A$, thus $A$ is $E_{c}$ (resp. $\delta$ - $\left.\beta_{\mathrm{c}}\right)$-open set, but it is not closed.

Remark 3.9: Every $E_{c}\left(\right.$ resp. $\delta$ - $\left.\beta_{c}\right)$-open is $E($ resp. $\delta$ - $\beta$ )open but the converse need not be true in general, the following Theorem shows that the family of $E \Sigma(X)$ (resp. $\delta-\beta \Sigma(X))$ is identical to the family of $E C \Sigma(X)$ (resp. $\delta-\beta C \Sigma(X))$.

Theorem 3.10: If a space $(X, T)$ is $T_{1}$-Space, then the families $E \Sigma(X)$ (resp. $\delta$ - $\beta \Sigma(X))$ are identical to the families $E C \Sigma(X)$ (resp. $\delta$ - $\beta C \Sigma(X))$ (i. e) $E \Sigma(X)$ (resp. $\delta$ $\beta \Sigma(X))=E C \Sigma(X)(\operatorname{resp} . \delta-\beta C \Sigma(X))$.

Proof: Let $(X, T)$ be a Topological space and $A$ be any subset of a space $X(s . t) A \in E \Sigma(X)(\operatorname{resp} . \delta-\beta \Sigma(X))$, there are two cases, if $A=\varphi$, so $A \in E C \Sigma(X)$ (resp. $\delta$ $\beta C \Sigma(X))$, if $A \neq \varphi$, Since a space $X$ is $T_{1}$, then every singleton is closed set and hence $\forall \varkappa \in A$, we get $\varkappa \in$ $\{\varkappa\} \subseteq A$. thus, $A \in E C \Sigma(X)(\operatorname{resp} . \delta$ - $\beta C \Sigma(X))$, so $E \Sigma(X)$ (resp. $\delta-\beta \Sigma(X)) \subseteq E C \Sigma(X) \quad($ resp. $\quad \delta-\beta C \Sigma(X))$, but generally, $E C \Sigma(X)$ (resp. $\delta-\beta C \Sigma(X)) \subseteq E \Sigma(X)$ (resp. $\delta$ $\beta \Sigma(X))$, therefore:

$E \Sigma(X)(\operatorname{resp} . \delta-\beta \Sigma(X))=E C \Sigma(X)(\operatorname{resp} . \delta-\beta C \Sigma(X))$.

Now we show that in any Topological space $(X, T)$ the arbitrary unions of $E_{c}$ (resp. $\delta$ - $\beta_{\mathrm{c}}$ )-open sets is $E_{c}$ (resp. $\delta$ - $\beta_{\mathrm{c}}$ )-open. 
Theorem 3.11: Let $(X, T)$ be a Topological space and $\left\{A_{\lambda}: \lambda \in \Delta\right\}$ be a family of $E_{c}$ (resp. $\delta$ - $\beta_{\mathrm{c}}$ )-open sets in a space $X$. Then $U\left\{A_{\lambda}: \lambda \in \Delta\right\}$ is $E_{c}\left(\right.$ resp. $\delta$ - $\left.\beta_{\mathrm{c}}\right)$-open.

Proof: suppose that $\left\{A_{\lambda}: \lambda \in \Delta\right\}$ be a collection of $E_{c}$ (resp. $\delta$ - $\beta_{\mathrm{c}}$ )-open sets $\forall \lambda \in \Delta$, then $A_{\lambda}$ is $E$ (resp. $\left.\delta-\beta\right)$ open sets $\forall \lambda$ and so via (Proposition 2.11), $\bigcup\left\{A_{\lambda}: \lambda \in\right.$ $\Delta\}$ is $E$ (resp. $\delta$ - $\beta$ )-open. If $\varkappa \in \bigcup\left\{A_{\lambda}: \lambda \in \Delta\right\}, \exists \lambda \in \Delta \exists$ $\mathcal{H} \in A_{\lambda}$. Since $A_{\lambda}$ is $E_{c}$ (resp. $\delta$ - $\beta_{\mathrm{c}}$ )-open $\forall \lambda \in \Delta, \exists$ a closed set $F \ni \varkappa \in F \subseteq A_{\lambda} \subseteq \bigcup_{\lambda \in \Delta} A_{\lambda} \Rightarrow \varkappa \in F \subseteq$ $\bigcup_{\lambda \in \Delta} A_{\lambda}$. so $\bigcup\left\{A_{\lambda}: \lambda \in \Delta\right\}$ is $E_{c}$ (resp. $\delta-\beta_{\mathrm{c}}$ )-open set.

Theorem 3.12: Let $(X, T)$ be a Topological space and $\left\{A_{\lambda}: \lambda \in \Delta\right\}$ be a family of $E_{c}$ (resp. $\delta$ - $\beta_{\mathrm{c}}$ )-closed sets in a space $X$. Then $\bigcap\left\{A_{\lambda}: \lambda \in \Delta\right\}$ is $E_{c}$ (resp. $\delta-\beta_{\mathrm{c}}$ )closed.

Proof: The proof is obvious it is follows from (Theorem 3.11) and using De Morgan's Law.

Remark 3.13: The intersection of two $E_{c}$ (resp. $\delta$ - $\beta_{\mathrm{c}}$ )open sets not necessary to be $E_{c}\left(\right.$ resp. $\delta$ - $\beta_{c}$ )-open. (See the following example)

Examples 3.14: conceder $X=\{\varkappa, y, w, z\}$ with the Topology $T=\{\varphi,\{\varkappa\},\{y\},\{x, y\},\{\varkappa, y, w\}, X\}$. Then the family of all closed subsets is: $T^{c}=\{X,\{y, w, z\},\{\varkappa$, $w, z\},\{w, z\},\{z\}, \varphi\}$. Thus: A subsets $A=\{\varkappa, w, z\}$ and $B=\{y, w, z\} \in E C \Sigma(X)($ resp. $\delta-\beta C \Sigma(X))$ but

$A \cap B=\{\mathrm{w}, \mathrm{z}\} \notin E C \Sigma(X)($ resp. $\delta-\beta C \Sigma(X))$.

Remark 3.15: It clears that from (example 3.14) the collection of all $\operatorname{EC} \Sigma(X)$ (resp. $\delta-\beta C \Sigma(X)$ ) is a supraTopology and not necessary to be a Topology in general.

The following Theorem explains the sufficient condition which makes the collection of all $E C \Sigma(X)$ (resp. $\delta-\beta C \Sigma(X)$ ) is Topology on $X$.

Theorem 3.16: If $E \Sigma(X)$-(resp. $\delta$ - $\beta \Sigma(X))$ of a space $X$ is a Topology on $X$, then $E C \Sigma(X)($ resp. $\delta$ - $\beta C \Sigma(X))$ is also a Topology on $X$

Proof: obvious $\varphi \& X \in E C \Sigma(X)$ (resp. $\delta-\beta C \Sigma(X))$, and via (Theorem 3.11), the union of any collection of $E C \Sigma(X)($ resp. $\delta$ - $\beta C \Sigma(X))$ is $E_{c}\left(\right.$ resp. $\delta$ - $\left.\beta_{\mathrm{c}}\right)$-open. Now we explain that the finite intersection of $E_{c}$ (resp. $\delta-\beta_{\mathrm{c}}$ )open sets is also $E_{c}$ (resp. $\delta$ - $\beta_{\mathrm{c}}$ )-open. Suppose that $A \&$ $B$ be two $E_{c}$ (resp. $\delta$ - $\beta_{\mathrm{c}}$ )-open sets $\Rightarrow A \& B$ are $E$-(resp. $\delta$ - $\beta$ )-open sets. Since $E \Sigma(X) \quad($ resp. $\delta-\beta \Sigma(X))$ is a Topology on $X$, hence $A \cap B \in E \Sigma(X)$ (resp. $\delta$ - $\beta \Sigma(X)$ ). Suppose that $\varkappa \in A \cap B \Rightarrow \varkappa \in A \& \mathcal{\varkappa} \in B$, thus $\exists$ closed sets $F \& E$ (s.t) $\varkappa \in F \subseteq A$ and $\varkappa \in E \subseteq B \Rightarrow$ $\varkappa \in F \cap E \subseteq A \cap B \Rightarrow A \cap B$ is $E_{c}\left(\right.$ resp. $\delta$ - $\beta_{\mathrm{c}}$ )-open sets.

Theorem 3.17: A subset $A$ of a space $(X, T)$ is $E_{c}$ (resp. $\delta$ - $\beta_{\mathrm{c}}$ )-open set iff $\forall \mathcal{X} \in A \exists$ a $E_{c}$ (resp. $\delta$ - $\beta_{\mathrm{c}}$ )-open set $B$ (s. t) $\varkappa \in B \subseteq A$.
Proof: The proof is obvious it's follows from the definition (3.2) and (Theorem 3.12).

Remark 3.18: If a space $(X, T)$ is $T_{1}$-Space, then $T \subseteq$ $E C \Sigma(X)($ resp. $\delta-\beta C \Sigma(X))$, since every open set is $\boldsymbol{E}$. $($ resp. $\boldsymbol{\delta}-\boldsymbol{\beta})$ )-open.

Theorem 3.19: Let $A$ be a sub set of a space $X$. if $A$ is a $\theta$-semi-open set. Then $A$ is $\delta$ - $\beta_{\mathrm{c}}$-open set.

Proof: suppose that $A$ is a $\theta$-semi-open in $X$, then $\forall \varkappa \in A \exists$ a semi-open set $G_{\varkappa}$ (s. t): $\varkappa \in G_{\varkappa} \subseteq \mathrm{Cl}\left(G_{\varkappa}\right) \subseteq A$, so $\bigcup_{\varkappa \in A}\{\varkappa\} \in \bigcup_{\varkappa \in A} G_{\varkappa} \subseteq$ $\bigcup_{\varkappa \in A} \mathrm{Cl}\left(G_{\varkappa}\right) \subseteq A \Rightarrow A=\bigcup_{\varkappa \in A} G_{\varkappa}$, which means $A$ is a union of semi-open sets and therefore $A$ is semi-open, thus $A$ is $\delta$ - $\beta$-open, as well $A=\mathrm{U}_{\varkappa \in A} \operatorname{Cl}\left(G_{\varkappa}\right)$ which is a union of closed sets, hence via Theorem (3.4) we get $A$ is $\delta$ - $\beta_{\mathrm{c}}$-open set.

The following example explains that the converse of Theorem (3.19) need not be true in general.

Example 3.20: because any space $X$ with the co-finite topology is $T_{1}$, in this case the family of $\delta-\beta \Sigma(X)$ is identical to the family of $\delta-\beta C \Sigma(X)$, thus any open set $G$ is $\boldsymbol{\delta}$ - $\boldsymbol{\beta}_{\text {copen }}$ but not $\theta$-semi- open set since $\bar{G}=X$, for each open subset $G$ of $X$.

Theorem 3.21: Let $A$ be a sub set of a space $X$. if $A$ is a $\theta$-open set. Then $A$ is $E_{c}\left(\right.$ resp. $\delta$ - $\beta_{\mathrm{c}}$ )-open set.

Proof: suppose that $A$ is $\theta$-open set in $X$, then $\forall \varkappa \in A \exists$ an open set $G_{\mathcal{\varkappa}}$ (s. t):

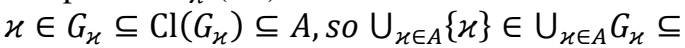
$\bigcup_{\mathcal{X} \in A} \mathrm{Cl}\left(G_{\mathcal{\varkappa}}\right) \subseteq A \Rightarrow A=\bigcup_{\mathcal{X} \in A} G_{\mathcal{H}}$, which means $A$ is a union of open sets and therefore $A$ is open set, thus $A$ is $E$ - $\left(\right.$ resp. $\delta$ - $\beta$ )-open, as well $A=\bigcup_{\varkappa \in A} \operatorname{Cl}\left(G_{\varkappa}\right)$ which is a union of closed sets, hence via Theorem (3.4) we get $A$ is $E_{c}\left(\right.$ resp. $\delta$ - $\beta_{\mathrm{c}}$ )-open set.

The following example explains that the converse of Theorem (3.21) need not be true in general.

Examples 3.22: because any space $X$ with the co-finite topology is $T_{1}$, so the families of $E \Sigma(X)($ resp. $\delta-\beta \Sigma(X))$ are identical to the families of $E C \Sigma(X)$ (resp. $\delta$ $\beta C \Sigma(X))$, therefore any open set $G$ is $E_{c}\left(\right.$ resp. $\delta$ - $\beta_{\mathrm{c}}$ )open but not $\theta$-open set since $\bar{G}=X$, For all open subset $G$ of $X$.

Theorem 3.23: Every regular closed subset in a space $(X, T)$ is $\delta-\beta_{\mathrm{c}}$-open set.

Proof: suppose that $A$ is regular closed subset in $X$, thus $A=C l(\operatorname{Int}(A))$, but $\mathrm{Cl}(\operatorname{Int}(A)) \subseteq C l(\operatorname{Int}(\delta-C l(A))) \Longrightarrow A$ is $\delta$ - $\beta$-open. Now, since $A$ is closed, then via Definition (3.2), $A$ is $\delta$ - $\beta_{\mathrm{c}}$-open set.

Theorem 3.24: If $X$ is locally indiscrete space, then every semi-open set is $\delta-\beta_{\mathrm{c}}$-open set. 
Proof: Assume that $A$ is semi-open subset in $X$, thus $A$ $\subseteq C l(\operatorname{Int}(A)) \subseteq C l(\operatorname{Int}(C l(A))) \subseteq C l(\operatorname{Int}(\delta-C l(A))) \Rightarrow A$ is $\delta$ - $\beta$-open set. Since $X$ is locally indiscrete $\Rightarrow \operatorname{Int}(A)$ is closed and $A \subseteq C l(\operatorname{Int}(A))=\operatorname{Int}(A) \Longrightarrow A$ is open set and $\forall \varkappa \in A \Longrightarrow \varkappa \in \operatorname{Int}(A) \subseteq A$. Thus, via Definition (3.2part-ii), we get $A$ is $\delta-\beta_{\mathrm{c}}$-open set.

Theorem 3.25: Let $X$ be a Topological space, if $X$ is Regular space, then every open set is a $E_{c}\left(\right.$ resp. $\delta$ - $\left.\beta_{\mathrm{c}}\right)$ open set.

Proof: suppose that $A$ is any open subset of $X$, so $A$ is $E$ - $($ resp. $\delta$ - $\beta$ )-open. There are two cases, if $A=\varphi$, thus $A \in E C \Sigma(X)$ (resp. $\delta$ - $\beta C \Sigma(X)$ ), if $A \neq \varphi$, since $X$ is regular, then via definition (2.9), $\forall \varkappa \in A \subseteq X \exists$ An open set $G$ (s. t), $\mathcal{x} \in G \subseteq C l(G) \subseteq A$. So, $\varkappa \in C l(G) \subseteq$ $A$. Therefore via definition (3.2), we have $T \in E C \Sigma(X)$ (resp. $\delta$ - $\beta C \Sigma(X)$ ).

We state in the next Theorem that the family of $\theta$-semiopen sets and the family of $\delta-\beta_{\mathrm{c}}$-open sets are identical.

Theorem 3.26: Let $(X, T)$ be a finite topological space, then every $\theta S \Sigma(X)=\delta-\beta C \Sigma(X)$.

Proof: we must prove that $\theta S \Sigma(X) \subseteq \delta-\beta C \Sigma(X) \& \delta$ $\beta C \Sigma(X) \subseteq \theta S \Sigma(X)$, we already proved that $\theta S \Sigma(X) \subseteq \delta$ $\beta C \Sigma(X)$ in Theorem (3.19), now we prove the other part, let $A \in \delta$ - $\beta C \Sigma(X)$, thus, $A$ is $\delta$ - $\beta$-open set, and via Theorem (3.4), $A=\bigcup F_{\lambda}$ where $F_{\lambda}$ closed set $\forall \lambda \in A$. Since $X$ is finite $\Longrightarrow \exists$ a closed set $F \in X$ (s. t) $F=$ $U F_{\lambda} \Rightarrow A=F \Rightarrow A$ is both $\delta$ - $\beta$-open set and closed. Thus $A \in \theta S \Sigma(X)$

Corollary 3.27: Let $(X, T)$ be an Alexandroff Topological space, then every $\theta S \Sigma(X)=\delta-\beta C \Sigma(X)$.

Proof: The proof is obvious it is follows from Theorem (3.26).

Theorem 3.28: Let $(X, T)$ be a finite topological space, then every $E_{c}\left(\right.$ resp. $\delta-\beta_{\mathrm{c}}$ )-open set is clopen set.

Proof: suppose $A \in E C \Sigma(X)$ (resp. $\delta-\beta C \Sigma(X)$ ), thus, $E_{\text {- }}$ (resp. $\delta$ - $\beta$ )-open set, and via Theorem (3.4), $A=U F_{\lambda}$ where $F_{\lambda}$ closed set $\forall \lambda \in A$. Since $X$ is finite $\Rightarrow \exists \mathrm{a}$ closed set $F \in X$ (s. t) $F=\bigcup F_{\lambda} \Rightarrow A=F \Rightarrow A$ is both $E$-(resp. $\delta$ - $\beta$ )-open set and closed sets. Therefore:

$A \subseteq C l(\delta$-Int $(A)) \cup \quad \operatorname{Int}(\delta-C l(A))($ resp. $\quad A \subseteq C l(\operatorname{Int}(\delta$ $C l(A)))$. Thus $A$ is clopen.

Corollary 3.29: Let $(X, T)$ be an Alexandroff Topological space, then every $E_{c}\left(\operatorname{resp} . \delta-\beta_{\mathrm{c}}\right)$-open set is clopen set.

Proof: The proof is clear it is follows directly from Theorem (3.28).

Theorem 3.30: The following statements hold for subsets $A$ and $B$ of an extremally disconnected Topological space $(X, T)$ :
I. If $A \in E C \Sigma(X)$ and $B$ a regular open. Then $A \cap B$ is a $E_{c}$-open set.

II. If $A \in \delta-\beta C \Sigma(X)$ and $B$ a regular open. Then $A \cap B$ is a $\delta$ - $\beta_{\text {c }}$-open set.

III. If $A \in \delta \Sigma(X)$. Then $A$ is a $E_{c}$-open set.

IV. If $A$ is a regular open subset of $X$. Then $A$ is a $E_{c}$-open set.

V. If $A \in \delta \Sigma(X)$. Then $A$ is a $\delta$ - $\beta_{\mathrm{c}}$-open set.

VI. If $A$ is a regular open subset of $X$. Then $A$ is a $\delta$ - $\beta_{\mathrm{c}}$-open set.

Proof: (i) - suppose that $X$ is an extremally disconnected topological space and $A, B \subseteq X$. Since $A$ is $E_{c}$-open and $B$ is a regular open then, $A$ is $E$-open and $B$ is open set, so we have:

$A \cap B \subseteq(C l(\delta-\operatorname{Int}(A)) \cup \operatorname{Int}(\delta-\operatorname{Cl}(A))) \cap B=$ $(C l(\delta-\operatorname{Int}(A)) \cap B) \cup(\operatorname{Int}(\delta-\operatorname{Cl}(A)) \cap B) \subseteq$ $(C l(\delta-\operatorname{Int}(A) \cap B)) \cup(\operatorname{Int}(\delta-\operatorname{Cl}(A) \cap B)) \subseteq$ $(C l(\delta-\operatorname{Int}(A \cap B))) \cup(\operatorname{Int}(\delta-C l(A \cap B)))$. Thus $A \cap B$ is $\boldsymbol{E}$-open set. Now let $\varkappa \in A \cap B$, implies $\varkappa \in A$ and $\varkappa \in B$ so, since $A$ is $E_{c}$-open $\Rightarrow \exists$ a closed set $F$ (s. t) $\varkappa \in F \subseteq A$ and hence, $\varkappa \in F \cap B \subseteq A \cap B$. since $B$ is regular open an extremally disconnected space, thus $B$ is closed and hence $F \cap B$ is closed set. Consequently $A \cap B$ is a $\boldsymbol{E}_{c}$-open set.

Proof: (ii)- Since $A$ is $\delta$ - $\beta_{\mathrm{c}}$-open and $B$ is a regular open then, $A$ is $\delta$ - $\beta$-open and $B$ is open set, so we have: $A \cap B \subseteq C l(\operatorname{Int}(\delta-C l(A))) \cap B \subseteq C l(\operatorname{Int}(\delta-$ $C l(A)) \cap B) \subseteq$ $C l(\operatorname{Int}(\delta-C l(A) \cap B)) \subseteq C l(\operatorname{Int}(\delta-C l(A \cap B)))$. Thus $A \cap B$ is $\delta$ - $\beta$-open set. Now let $\varkappa \in A \cap B$, implies $\varkappa \in A$ and $\varkappa \in B$ so, since $A$ is $\delta-\beta_{\text {copen }} \Rightarrow \exists$ a closed set $F$ (s. t) $\varkappa \in F \subseteq A$ thus, $\varkappa \in F \cap B \subseteq$ $A \cap B$. since $B$ is regular open an extremally disconnected space, hence $B$ is closed and so $F \cap B$ is closed set. Therefore $A \cap B$ is a $\boldsymbol{\delta}-\boldsymbol{\beta}_{\mathbf{c}}$-open set.

Proof: (iii)- suppose that $X$ is an extremally disconnected topological space and $A \in \delta \Sigma(X)$. Then $\forall \varkappa \in A \exists$ an open set $\mathrm{G}_{\varkappa}$ (s. t): $\mathcal{X} \in \mathrm{G}_{\mathcal{\varkappa}} \subseteq \operatorname{Int}\left(\mathrm{Cl}\left(\mathrm{G}_{\varkappa}\right)\right) \subseteq \mathrm{A} \Rightarrow \mathrm{U}_{\mathcal{X} \in \mathrm{A}} G_{\mathcal{\varkappa}}=A$ is an open set, so $A$ is a $E$-open. Since $X$ is extremally disconnected space, hence $\operatorname{Int}\left(\operatorname{Cl}\left(G_{\mathcal{\varkappa}}\right)\right)=\operatorname{Cl}\left(G_{\mathcal{\varkappa}}\right)$ and $\mathcal{X} \in C l\left(G_{\mathcal{\varkappa}}\right) \subseteq A$. thus $A$ is a $E_{c}$-open set.

Proof: (iv)- The proof follows immediately from Theorem (3.30-part(iii)) and the fact that $R \Sigma(X) \subseteq$ $\boldsymbol{\delta} \Sigma(X)$.

Proof: (v)- suppose that $X$ is an extremally disconnected topological space and $A \in \delta \Sigma(X)$. Then $\forall \varkappa \in A \exists$ an open set $G_{\varkappa}$ (s. t): $\varkappa \in G_{\varkappa} \subseteq \operatorname{Int}\left(C l\left(G_{\varkappa}\right)\right) \subseteq A \Rightarrow \bigcup_{\varkappa \in A} G_{\varkappa}=A \quad$ is $\quad$ an open set, so $A$ is a $\delta$ - $\beta$-open. Since $X$ is extremally disconnected, consequently $\operatorname{Int}\left(\mathrm{Cl}\left(G_{\varkappa}\right)\right)=\operatorname{Cl}\left(G_{\mathcal{\varkappa}}\right)$ and $\mathcal{X} \in \operatorname{Cl}\left(G_{\mathcal{\varkappa}}\right) \subseteq A$. Thus $A$ is a $\delta-\beta_{\text {c }}$-open set. 
Proof: (vi) - This proof follows directly from Theorem (3.30-part (v)) and the fact that $R \Sigma(X) \subseteq \delta \Sigma(X)$.

Theorem 3.31: Let $(X, T)$ be a Topological space and $A, B \subseteq X$. if $A \in E C \Sigma(X)$ (resp. $A \in \delta-\beta C \Sigma(X)$ ), and $B$ is $\delta$-clopen, then, $A \cap B \in E C \Sigma(X)(\operatorname{resp} . A \cap B \in \boldsymbol{\delta}-$ $ß C \Sigma(X))$.

Proof: suppose $A \in E C \Sigma(X)$ (resp. $A \in \delta$ - $\beta C \Sigma(X)$ ), and $B$ is $\delta$-clopen, thus $A$ is $E$ - $($ resp. $\delta$ - $\beta$ )-open and $B$ is $\delta$ open, so via Lemma (2.13) we get $A \cap B \in$ $\boldsymbol{E} \Sigma(X)($ resp. $A \cap B \in \boldsymbol{\delta}-\beta \Sigma(X))$. Now let $\quad \varkappa \in$ $A \cap B$, implies $\varkappa \in A$ and $\varkappa \in B$ so, since $A$ is $E_{c}$ (resp. $\delta$ - $\beta_{\mathrm{c}}$ )-open $\Rightarrow \exists$ a closed set $F$ (s. t) $\mathcal{X} \in F \subseteq A$ thus, $\varkappa \in F \cap B \subseteq A \cap B$. since $B$ is $\delta$-clopen, hence $B$ is $\delta$ closed and consequently $B$ is closed set $\Rightarrow F \cap B$ is closed set. Therefore $A \cap B \in \boldsymbol{E} \boldsymbol{C} \Sigma(X)($ resp. $A \cap B \in$ $\boldsymbol{\delta}-\beta C \Sigma(X))$.

Theorem 3.32: Let $(X, T)$ be a Topological space and $A, B \subseteq X$. if $A \in E C \Sigma(X)$ (resp. $A \in \delta-\beta C \Sigma(X)$ ), and $B$ is $\theta$-open and closed, then, $A \cap B \in \boldsymbol{E} \boldsymbol{C} \Sigma(X)($ resp. $A \cap B \in \boldsymbol{\delta}-\beta C \Sigma(X))$.

Proof: The proof is similar to that of Theorem (3.31).

Remarks 3.33 For a subset $A$ of a space $X$, we have the following results:

i) Since semi-open set $\Rightarrow$ b-open set $\Rightarrow \beta$-open set $\Rightarrow \delta$ - $\beta$-open set, then:

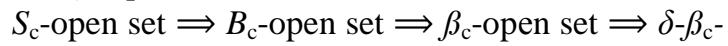
open set.

ii) Since pre-open set $\Rightarrow$ b-open set $\Rightarrow \beta$-open set $\Rightarrow$ $\delta$ - $\beta$-open set, then:

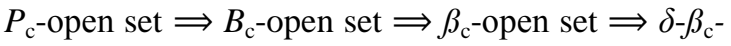
open set.

iii) Since pre-open $\Rightarrow E$-open $\Rightarrow \delta$ - $\beta$-open set, then: $P_{\mathrm{c}}$-open $\Rightarrow E_{\mathrm{c}}$-open set $\Rightarrow \delta$ - $\beta_{\mathrm{c}}$-open set.

Remarks (3.33) imply the following results:

Theorem 3.34: Let $(X, T)$ be a Topological space, then $S C \Sigma(X) \cup P C \Sigma(X) \subseteq B C \Sigma(X) \subseteq \beta C \Sigma(X) \subseteq \delta-\beta C \Sigma(X)$.

Theorem 3.35: Let $(X, T)$ be an Alexandroff Topological space, $S C \Sigma(X)=B C \Sigma(X)=\beta C \Sigma(X)=\delta$ $\beta C \Sigma(X)$.

Proof: Via Theorem (3.34), we have $S C \Sigma(X) \subseteq$ $B C \Sigma(X) \subseteq \beta C \Sigma(X) \subseteq \delta-\beta C \Sigma(X)$. If $A \in \delta-\beta C \Sigma(X)$, then $A$ is a union of closed sets in an Alexandroff space $\Rightarrow A$ is closed. Thus we get $A \subseteq C l(\operatorname{Int}(\delta-C l(A))) \subseteq$ $C l(\operatorname{Int}(C l(A)))=C l(\operatorname{Int}(A)) \Longrightarrow \mathrm{A}$ is semi-open set. So $A \in S C \Sigma(X)$, therefore $S C \Sigma(X) \subseteq B C \Sigma(X) \subseteq \beta C \Sigma(X) \subseteq$ $\delta-\beta C \Sigma(X) \subseteq S C \Sigma(X)$ consequently $S C \Sigma(X)=B C \Sigma(X)=$ $\beta C \Sigma(X)=\delta-\beta C \Sigma(X)$.

Theorem 3.36: Let $(X, T)$ be a topological space and $A \subseteq X$. If $A$ is a clopen set, then $A \in E C \Sigma(X)$ (resp. $A \in$ $\boldsymbol{\delta}-\boldsymbol{\beta} C \Sigma(X))$.
Proof: suppose that $X$ is a topological space and $A \subseteq X$ where $A$ is a clopen set. Then $A$ is open so it is $E$-(resp. $\delta$ - $\beta$ )-open and $A$ is closed so it is a union of closed sets and hence, it is $E_{c}$ (resp. $\delta$ - $\beta_{\mathrm{c}}$ )-open. Therefore, $A \in$ $E C \Sigma(X)($ resp. $A \in \delta-\beta C \Sigma(X))$.

Theorem 3.37: The following statements are equivalent for a subset $A$ of a space $(X, T)$ :

I. $A$ is clopen set.

II. $\quad A$ is $E_{c}$ (resp. $\delta$ - $\beta_{\mathrm{c}}$ )-open set and closed.

III. $A$ is $E$-(resp. $\delta$ - $\beta$ )-open set and closed.

Proof: The proofs are obvious thus omitted.

Remarks 3.38: From Figure (2) we can notice the following facts in any space $(X, T)$

I. $T$-open sets is incomparable with $E C \Sigma(X)$ (resp. $\delta$ - $\beta C \Sigma(X))$.

II. $\quad \alpha \Sigma(X, T)$ is incomparable with $E C \Sigma(X)$ (resp. $\delta-\beta C \Sigma(X))$.

III. $\delta \Sigma(X, T)$ is incomparable with $E C \Sigma(X)$ (resp. $\delta-\beta C \Sigma(X))$.

IV. $\delta P \Sigma(X, T)$ is incomparable with $E C \Sigma(X)$ (resp. $\delta-\beta C \Sigma(X))$.

V. $P \Sigma(X, T)$ is incomparable with $E C \Sigma(X)$ (resp. $\delta-\beta C \Sigma(X))$.

VI. $R \Sigma(X, T)$ is incomparable with $E C \Sigma(X)$ (resp. $\delta-\beta C \Sigma(X))$.

VII. $\quad \theta S \Sigma(X, T)$ is incomparable with $E C \Sigma(X)$.

VIII. $\quad S \Sigma(X, T)$ is incomparable with $E C \Sigma(X)$.

IX. $\quad B \Sigma(X)$ is incomparable with $E C \Sigma(X)$.

X. $\quad \beta \Sigma(X)$ is incomparable with $E C \Sigma(X)$.

Theorem 3.39: let $\left(Y, T_{Y}\right)$ be a subspace of a space $X$, if $A \in E C \Sigma(X)$ (resp. $A \in \delta-\beta C \Sigma(X))$ and $A \subseteq Y$ (s. t) $Y$ is $E$-(resp. $\delta$ - $\beta$ )-open, then $A \in E C \Sigma(Y)$ (resp. $A \in \delta$ $\beta C \Sigma(Y))$

Proof: Let $A \in E C \Sigma(X)$ (resp. $\delta$ - $\beta C \Sigma(X)) \Rightarrow A \in E \Sigma(X)$ (resp. $\delta-\beta \Sigma(X))$. Since $A \subseteq Y$ and $Y$ is $E$ - $($ resp. $\delta$ - $\beta$ )open, thus via Lemma (2.14) $A$ is $E$-(resp. $\delta$ - $\beta$ )-open in subspace $Y$, as well:

$\forall \varkappa \in A \exists$ a closed set $F \in X$ (s. $t) \quad \mathcal{H} \in F \subseteq A$. Since $A \subseteq Y \Rightarrow F$ is closed in subspace $Y$. Hence $A \in E C \Sigma(Y)$ (resp. $A \in \delta-\beta C \Sigma(Y)$ )

Theorem 3.40: let $\left(Y, T_{Y}\right)$ be a subspace of a space $X$, if $A \in E C \Sigma(Y)$ (resp. $A \in \delta$ - $\beta C \Sigma(Y)$ ) and $A \subseteq Y$ and $Y$ is clopen, then $A \in E C \Sigma(X)$ (resp. $A \in \delta-\beta C \Sigma(X))$

Proof: Let $A \in E C \Sigma(Y)$ (resp. $\delta$ - $\beta C \Sigma(Y)) \Rightarrow A \in E \Sigma(Y)$ (resp. $\delta$ - $\beta \Sigma(Y)$ ), and $\forall \varkappa \in A \exists$ a closed set $F \in Y$ (s. $t)$ $\mathcal{u} \in F \subseteq A$. Since $Y$ is clopen $\Rightarrow Y$ is $E \Sigma(X)$ (resp. $\delta$ $\beta \Sigma(X))$ and since $A \in E \Sigma(Y)($ resp. $\delta-\beta \Sigma(Y))$, then via Lemma (2.14), $A \in E \Sigma(X)$ (resp. $\delta$ - $\beta \Sigma(X)$ ). Moreover since $Y$ is clopen $\Rightarrow Y$ is closed in $X$ and since $F$ is closed in $Y$, hence via Theorem (2.16) $F$ is closed set in $X$. So $A \in E C \Sigma(X)($ resp. $A \in \delta-\beta C \Sigma(X))$ 
Corollary 3.41: Let $A$ and $Y$ be any subsets of a space $X$ (s. t) $A \subseteq Y \subseteq X$ and $Y$ is clopen. Then $A \in E C \Sigma(Y)$ (resp. $A \in \delta-\beta C \Sigma(Y)$ ) iff $A \in E C \Sigma(X)$ (resp. $A \in \delta$ $\beta C \Sigma(X))$

Proof: The proof follows from Theorems (3.39) and (3.40).

Corollary 3.42: Let $A$ and $Y$ be any subsets of a space $X$. if $A \in E C \Sigma(X)$ (resp. $A \in \delta-\beta C \Sigma(X)$ ) and $Y$ is $\delta$ clopen subset of $X$. Then $A \cap Y \in E C \Sigma(Y)($ resp. $A \cap Y \in \boldsymbol{\delta}-\beta C \Sigma(Y))$.

Proof: Let $A \in E C \Sigma(X)$ (resp. $\delta-\beta C \Sigma(X)$ ) and $Y$ is $\delta$ clopen of $X \Rightarrow A \in E \Sigma(X)$ (resp. $\delta-\beta \Sigma(X)$ ) and $Y$ is $\delta$ open and $\delta$-closed of $X$, so via Lemma (2.13), $A \cap Y \in$ $\boldsymbol{E} \Sigma(X)($ resp. $A \cap Y \in \boldsymbol{\delta}-\beta \Sigma(X))$.

Since $A \in E C \Sigma(X)$ (resp. $\delta$ - $\beta C \Sigma(X)) \Rightarrow \forall \varkappa \in A \exists$ a closed set $F$ in $X$ (s. t) $\varkappa \in F \subseteq A$ thus, $\varkappa \in F \cap Y \subseteq$ $A \cap Y \Rightarrow A \cap Y \in E C \Sigma(X)($ resp. $A \cap Y \in \delta-$ $\beta C \Sigma(X))$ (s. t), $A \cap Y \subseteq Y$. Hence via Theorem (3.39) $A \cap Y \in \boldsymbol{E C C} \Sigma(Y)($ resp. $A \cap Y \in \boldsymbol{\delta}-\beta C \Sigma(Y))$.

Theorem 3.43: Let $\left(X, T_{X}\right)$ and $\left(Y, T_{Y}\right)$ be Topological spaces and $X \times Y$ be the product Topology, if $A \in$ $E C \Sigma(X)$ (resp. $A \in \delta-\beta C \Sigma(X))$ and $B \in E C \Sigma(Y)$ (resp. $A$ $\in \delta$ - $\beta C \Sigma(Y))$ then, $A \times B \in E C \Sigma(X \times Y)($ resp. $A \times B \in$ $\delta-\beta C \Sigma(X \times Y))$.

Proof: suppose that $(\varkappa, y) \in A \times B \Rightarrow \varkappa \in A \& y \in B$. Since $A \in E C \Sigma(X) \quad($ resp. $\quad \delta-\beta C \Sigma(X)) \Longrightarrow A \in E \Sigma(X)$ (resp. $\delta-\beta \Sigma(X)) \Longrightarrow \forall \varkappa \in A \exists$ a closed set $F$ in $X$ (s. t) $\varkappa \in F \subseteq A$.

Since $B \in E C \Sigma(Y) \quad$ (resp. $\delta-\beta C \Sigma(Y)) \Rightarrow B \in E \Sigma(Y)$ (resp. $\delta-\beta \Sigma(Y)) \Longrightarrow \forall y \in B \exists$ a closed set $E$ in $Y$ (s. t) $y \in E \subseteq B$. So $(\varkappa, y) \in F \times E \subseteq A \times B$, and via Lemma (2.15) $A \times B \in E \Sigma(X \times Y)$ (resp. $A \times B \in \delta$ $\beta \Sigma(X \times Y))$. Since $F \& E$ are closed in spaces $X$ and $Y$ respectively, we get $F \times E$ is closed in $X \times Y$. Thus $A \times B \in E C \Sigma(X \times Y)($ resp. $A \times B \in \delta-\beta C \Sigma(X \times Y))$.

\section{SOME FUNDAMENTAL PROPERTIES OF $E_{c}(\delta$ - $\beta_{\mathrm{c}}$ )-OPEN SETS}

In this part, several topological properties concerning of $E_{c}\left(\right.$ resp. $\delta$ - $\left.\beta_{\mathrm{c}}\right)$-Neighborhood, $E_{c}$ (resp. $\delta$ - $\beta_{\mathrm{c}}$ )-Interior, $E_{c}\left(\right.$ resp. $\delta$ - $\left.\beta_{\mathrm{c}}\right)$-Closure, $E_{c}\left(\right.$ resp. $\delta$ - $\left.\beta_{\mathrm{c}}\right)$-Derived and $E_{c}$ (resp. $\delta$ - $\beta_{\mathrm{c}}$ )-Frontier of a sets via the notions of $E_{\mathrm{c}}$-open and $\delta-\beta_{c}$-open sets that are similar to those of open sets are given.

Remark 4.1: The topological properties of $E_{c}$ (resp. $\delta$ $\beta_{\mathrm{c}}$ )-Neighborhood, $E_{c}$ (resp. $\delta$ - $\beta_{\mathrm{c}}$ )-Interior, $E_{c}$ (resp. $\delta$ $\beta_{\mathrm{c}}$ )-Closure, $E_{c}$ (resp. $\delta$ - $\beta_{\mathrm{c}}$ )-Derived and $E_{c}\left(\right.$ resp. $\delta$ - $\left.\beta_{\mathrm{c}}\right)$ Frontier are the same as in the supra-Topology, therefore the proofs of the following properties are obvious and they are follow from their respective definitions thus omitted.
Definition 4.2: Let $(X, T)$ be a Topological space. A subset $U_{x}$ of $X$ is said to be $E_{c}$ (resp. $\left.\delta-\beta_{\mathrm{c}}\right)$ Neighborhood of a point $\mathcal{\varkappa} \in X$ if $\exists E_{c}$ (resp. $\delta$ - $\beta_{\mathrm{c}}$ )open set $V$ in $X$ such that $x \in V \subseteq U_{x}$.

Remarks 4.3: Every $E_{c}\left(\right.$ resp. $\left.\delta-\beta_{\mathrm{c}}\right)$-Neighborhood of $\varkappa \in X$ is $E$ (resp. $\delta$ - $\beta$ )-Neighborhood, it follows from the fact every $E_{c}\left(\right.$ resp. $\delta$ - $\left.\beta_{\mathrm{c}}\right)$-open set is $E($ resp. $\delta$ - $\beta$ )-open.

Theorem 4.4: For every two subsets $A, B$ of a space $X$ and $A \subseteq B$, if $A$ is a $E_{c}$ (resp. $\delta-\beta_{\mathrm{c}}$ )-Neighborhood of a point $\mathcal{X} \in X$, then $B$ is $E_{c}$ (resp. $\delta-\beta_{\mathrm{c}}$ )-Neighborhood of a point $\mathcal{x} \in X$.

Definition 4.5: Let $(X, T)$ be a Topological space. A point $\mathcal{U} \in X$ is said to be $E_{c}$ (resp. $\delta$ - $\beta_{\mathrm{c}}$ )-Interior point of a set $A \subseteq X$ if $\exists E_{c}$ (resp. $\delta$ - $\beta_{\mathrm{c}}$ )-open set $V$ in $X$ such that $x \in V \subseteq A$. The set of all $E_{c}$ (resp. $\delta$ - $\beta_{\mathrm{c}}$ )-Interior points of $A$ is called $E_{c}$ (resp. $\delta$ - $\beta_{\mathrm{c}}$ )-Interior of $A$ and is denoted by $E_{c} \operatorname{Int}(A)\left(\right.$ resp. $\delta$ - $\left.\beta_{\mathrm{c}} \operatorname{Int}(A)\right)$.

Several properties concerning of the $E_{c}$ (resp. $\delta-\beta_{\mathrm{c}}$ )Interior of set are introduced in the following Theorem.

Theorem 4.6: Let $(X, T)$ be a Topological space. For subsets $A$ and $B$ of $X$ the following are hold:

i. $\quad E_{c} \operatorname{Int}(A)\left(\operatorname{resp} . \delta-\beta_{\mathrm{c}} \operatorname{Int}(A)\right)$ is the union of all $E_{c}\left(\right.$ resp. $\left.\delta-\beta_{\mathrm{c}}\right)$-open sets which are contained in A.

ii. $\quad E_{c} \operatorname{Int}(A)\left(\right.$ resp. $\delta$ - $\left.\beta_{\mathrm{c}} \operatorname{Int}(A)\right)$ is $E_{c}\left(\right.$ resp. $\left.\delta-\beta_{\mathrm{c}}\right)$ open set in $X$.

iii. $\quad A$ is $E_{c}\left(\right.$ resp. $\delta$ - $\left.\beta_{\mathrm{c}}\right)$-open set $\Leftrightarrow A=E_{c} \operatorname{Int}(A)$ (resp. $\left.\delta-\beta_{\mathrm{c}} \operatorname{Int}(A)\right)$.

iv. $\quad E_{c} \operatorname{Int}\left(E_{c} \operatorname{Int}(A)\right)\left(\operatorname{resp} . \quad \delta-\beta_{\mathrm{c}} \operatorname{Int}\left(\delta-\beta_{\mathrm{c}} \operatorname{Int}(A)\right)=\right.$ $E_{c} \operatorname{Int}(A)\left(\operatorname{resp} . \delta-\beta_{\mathrm{c}} \operatorname{Int}(A)\right)$.

v. $\quad E_{c} \operatorname{Int}(\varphi)\left(\operatorname{resp} . \delta-\beta_{\mathrm{c}} \operatorname{Int}(\varphi)\right)=\varphi$ and $E_{c} \operatorname{Int}(X)$ $\left(\right.$ resp. $\left.\delta-\beta_{\mathrm{c}} \operatorname{Int}(X)\right)=X$.

vi. $\quad E_{c} \operatorname{Int}(A)\left(\operatorname{resp} . \delta-\beta_{\mathrm{c}} \operatorname{Int}(A)\right) \subseteq A$.

vii. If $A \subseteq B$, then $E_{c} \operatorname{Int}(A)\left(\operatorname{resp} . \delta-\beta_{\mathrm{c}} \operatorname{Int}(A)\right) \subseteq$ $E_{c} \operatorname{Int}(B)\left(\right.$ resp. $\left.\delta-\beta_{\mathrm{c}} \operatorname{Int}(B)\right)$.

viii. $\quad E_{c} \operatorname{Int}(A)\left(\right.$ resp. $\delta$ - $\left.\beta_{\mathrm{c}} \operatorname{Int}(A)\right) \cup E_{c} \operatorname{Int}(B)($ resp. $\delta$ $\left.\beta_{\mathrm{c}} \operatorname{Int}(B)\right) \subseteq E_{c} \operatorname{Int}(A \cup B) \quad\left(\right.$ resp. $\quad \delta-\beta_{\mathrm{c}} \operatorname{Int}(A \cup$ $B))$.

ix. If $A \cap B=\varphi \Rightarrow E_{c} \operatorname{Int}(A)\left(\right.$ resp. $\left.\delta-\beta_{\mathrm{c}} \operatorname{Int}(A)\right) \cap$ $E_{c} \operatorname{Int}(B)\left(\right.$ resp. $\delta$ - $\left.\beta_{\mathrm{c}} \operatorname{Int}(B)\right)=\varphi$.

X. $\quad E_{c}$ Int $(A \cap B) \quad\left(\right.$ resp. $\quad \delta$ - $\beta_{\mathrm{c}} \operatorname{Int}(A \cap B) \subseteq$ $E_{c} \operatorname{Int}(A) \cap \quad E_{c} \operatorname{Int}(B)\left(\right.$ resp. $\quad \delta-\beta_{\mathrm{c}} \operatorname{Int}(A) \cap \quad \delta$ $\left.\beta_{\mathrm{c}} \operatorname{Int}(B)\right)$.

Definition 4.7: Let $(X, T)$ be a Topological space and $A \subseteq X$. The $E_{c}$ (resp. $\delta-\beta_{\mathrm{c}}$ )-Closure of $A$ in $X$ is the set, $E_{c-} C l(A)\left(\right.$ resp. $\left.\delta-\beta_{\mathrm{c}-} C l(A)\right)=\cap\left\{H \subseteq X: H\right.$ is $E_{c}$ (resp. $\delta$ $\beta_{\mathrm{c}}$ )-closed set and $\left.A \subseteq H\right\}$.

Theorem 4.8: Let $A$ be a subset of a space $X$. Then, $E_{c-}$ $C l(A)\left(\right.$ resp. $\delta$ - $\left.\beta_{\mathrm{c}-} C l(A)\right)$ is the smallest $E_{c}\left(\right.$ resp. $\left.\delta-\beta_{\mathrm{c}}\right)$ closed set containing $A$.

Theorem 4.9: $A$ subset $A$ of a space $X$ is $E_{c}$ (resp. $\delta$ - $\beta_{\mathrm{c}}$ )closed iff $E_{c-} C l(A)\left(\right.$ resp. $\left.\delta-\beta_{\mathrm{c}-} C l(A)\right)=A$. 
Theorem 4.10: For a subset $A$ of a space $X$ and $\varkappa \in X$. The following are equivalent

i. $\quad$ For any $E_{c}$ (resp. $\delta$ - $\beta_{\mathrm{c}}$ )-open set $U$ in $X$ such that $\varkappa \in U$ we get, $A \cap U \neq \varphi$.

ii. $\quad \varkappa \in E_{c-} C l(A)\left(\right.$ resp. $\delta$ - $\left.\beta_{\mathrm{c}-} C l(A)\right)$.

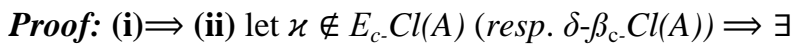

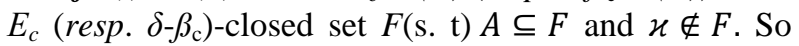
$\mathcal{u} \in X \backslash F$ and $X \backslash F$ is $E_{c}$ (resp. $\delta$ - $\beta_{\mathrm{c}}$ )-open set and therefore, $(X \backslash F) \cap A \subseteq(X \backslash F) \cap F=\varphi$. Which is a contradiction and therefore, $\varkappa \in E_{c-} C l(A)$ (resp. $\delta-\beta_{c \text { - }}$ $C l(A))$.

(ii) $\Rightarrow$ (i) Assume that there exists $E_{c}$ (resp. $\delta$ - $\beta_{\mathrm{c}}$ )-open set $\mathrm{U}$ containing $x$ with $A \cap U=\varphi$. Then $A \subseteq(X / U)$ and $X / U$ is $E_{c}$ (resp. $\delta$ - $\beta_{\text {c }}$ )-closed set. Hence $\varkappa \notin E_{c \text { - }}$ $C l(A)\left(\right.$ resp. $\left.\delta-\beta_{\mathrm{c}-} C l(A)\right)$, which is a contradiction.

Some properties of $E_{c}\left(\right.$ resp. $\left.\delta-\beta_{\mathrm{c}}\right)$-closure of sets are introduced in the following Theorem.

Theorem 4.11: Let $(X, T)$ be a Topological space. For subsets $A \& B$ of $X$ the following properties hold:

i. $A \subseteq E_{c-} C l(A)\left(\right.$ resp. $\left.A \subseteq \delta-\beta_{\mathrm{c}_{-}} C l(A)\right)$.

ii. $E_{c-} C l(\varphi)\left(\right.$ resp. $\left.\delta-\beta_{\mathrm{c}-} C l(\varphi)\right)=\varphi$ and $E_{c-} C l(X)$ (resp. $\left.\delta-\beta_{\mathrm{c}-} C l(X)\right)=X$.

iii. If $A \subseteq B$, then $E_{c-} C l(A)\left(\right.$ resp. $\left.\delta-\beta_{\mathrm{c}-} C l(A)\right) \subseteq E_{c \text { - }}$ $C l(B)\left(\right.$ resp. $\left.\delta-\beta_{\mathrm{c}-} C l(B)\right)$.

iv. $\quad E_{c-} C l\left(E_{c-} C l(A)\right)\left(\right.$ resp. $\delta-\beta_{\mathrm{c}-} C l\left(\delta-\beta_{\mathrm{c}-} C l(A)\right)=E_{c-}$ $C l(A)\left(\right.$ resp. $\left.\delta-\beta_{\mathrm{c}-} C l(A)\right)$.

v. If $E_{c-} C l(A)\left(\right.$ resp. $\left.\delta-\beta_{\mathrm{c}-} C l(A)\right) \cap E_{c-} C l(B)$ (resp. $\left.\delta-\beta_{\mathrm{c}-} C l(B)\right)=\varphi, \Longrightarrow A \cap B=\varphi$.

vi. $\quad E_{c-} C l(A)\left(\right.$ resp. $\left.\delta-\beta_{\mathrm{c}-} C l(A)\right) \cup E_{c-} C l(B)($ resp. $\delta$ $\left.\beta_{\mathrm{c}-} C l(B)\right) \subseteq E_{c-} C l(A \cup B)\left(\right.$ resp. $\delta-\beta_{\mathrm{c}-} C l(A \cup$ $B))$.

vii. $\quad E_{c-} C l(A \cap B) \quad\left(\right.$ resp. $\quad \delta-\beta_{c-} C l(A \cap B) \subseteq E_{c-}$ $C l(A) \cap \quad E_{c-} C l(B)\left(\right.$ resp. $\quad \delta-\beta_{\mathrm{c}-} C l(A) \cap \quad \delta-\beta_{\mathrm{c}-}$ $C l(B))$.

The relations between the $E_{c}$ (resp. $\delta-\beta_{c}$ )closure and $E_{c}$ (resp. $\delta$ - $\beta_{\mathrm{c}}$ )-Interior for a subset $A$ of $X$ can be considered in the following Theorem.

Theorem 4.12: Let $(X, T)$ be a topological space. For subset $A$ of $X$ the following statements hold:

I. $\quad\left[E_{c-} C l(A)\left(\text { resp. } \delta-\beta_{\mathrm{c}-} C l(A)\right)\right]^{\mathrm{c}}=E_{c} \operatorname{Int}\left(A^{\mathrm{c}}\right)($ resp. $\left.\delta-\beta_{\mathrm{c}} \operatorname{Int}\left(A^{\mathrm{c}}\right)\right)$.

II. $\quad\left[E_{c} \operatorname{Int}(A)\left(\operatorname{resp} . \delta-\beta_{\mathrm{c}} \operatorname{Int}(A)\right)\right]^{\mathrm{c}}=E_{c-} C l\left(A^{\mathrm{c}}\right)(\operatorname{resp}$. $\left.\delta-\beta_{\mathrm{c}-} C l\left(A^{c}\right)\right)$.

III. $\quad E_{c-} C l(A)\left(\right.$ resp. $\left.\delta-\beta_{\mathrm{c}-} C l(A)\right)=\left[E_{c} \operatorname{Int}\left(A^{c}\right)(\right.$ resp. $\left.\left.\delta-\beta_{\mathrm{c}} \operatorname{Int}\left(A^{c}\right)\right)\right]^{\mathrm{c}}$.

IV. $\quad E_{c} \operatorname{Int}(A)\left(\right.$ resp. $\left.\delta-\beta_{\mathrm{c}} \operatorname{Int}(A)\right)=\left[E_{c-} C l\left(A^{c}\right)(\right.$ resp. $\left.\left.\delta-\beta_{\mathrm{c}-} \mathrm{Cl}\left(A^{c}\right)\right)\right]^{\mathrm{c}}$.

Definition 4.13: Let $A$ be a subset of a space $X$. A point $\varkappa \in X$ is said to be $E_{c}$ (resp. $\delta$ - $\beta_{\mathrm{c}}$ )-Limit point of $A$ if for each $E_{c}$ (resp. $\delta$ - $\beta_{\mathrm{c}}$ )-open subset $U$ of $X$ containing $x$, $U \cap A \backslash\{x\} \neq \varphi$. the set of all $E_{c}$ (resp. $\delta$ - $\beta_{\mathrm{c}}$ )-Limit points of $A$ is called the $E_{c}$ (resp. $\delta$ - $\beta_{\mathrm{c}}$ )-Derived set of $A$ and its denoted by $E_{c} D(A)\left(\right.$ resp. $\left.\delta-\beta_{\mathrm{c}} D(A)\right)$.
Proposition4.14: Let $A$ be a subset of a space $X$. If for each closed set $F \subseteq X$ containing $x$ satisfies $F \cap$ $(A \backslash\{x\}) \neq \varphi$. then the point $\varkappa \in X$ is $E_{c}$ (resp. $\delta$ - $\beta_{\mathrm{c}}$ )Limit point of $A$.

Several properties concerning of the $E_{c}$ (resp. $\delta$ - $\beta_{\mathrm{c}}$ )Derived set are explained in the following Theorem.

Theorem 4.15: For any subsets $A$ and $B$ of a space $X$ the following properties hold:

I. If $A \subseteq B$, then $E_{c-} D(A)\left(\right.$ resp. $\left.\delta-\beta_{\mathrm{c}-} D(A)\right) \subseteq E_{c-}$ $D(B)\left(\right.$ resp. $\left.\delta-\beta_{\mathrm{c}} D(B)\right)$.

II. $\quad E_{c-} D(\varphi)\left(\right.$ resp. $\left.\delta-\beta_{\mathrm{c}-} D(\varphi)\right)=\varphi$.

III. If $\varkappa \in E_{c} D(A)$ (resp. $\left.\delta-\beta_{\mathrm{c}} D(A)\right) \Rightarrow \varkappa \in E_{c}$ $D(A \backslash\{x\})\left(\right.$ resp. $\left.\delta-\beta_{\mathrm{c}} . D(A \backslash\{x\})\right)$.

IV. $\quad E_{c} D(A)\left(\right.$ resp. $\left.\delta-\beta_{\mathrm{c}} D(A)\right) \cup E_{c} D(B)\left(\right.$ resp. $\delta-\beta_{\mathrm{c}}$ $D(B)) \subseteq E_{c-} D(A \cup B)\left(\right.$ resp. $\left.\delta-\beta_{\mathrm{c}-} D(A \cup B)\right)$.

V. $\quad E_{c-} D(A \cap B) \quad\left(\right.$ resp. $\quad \delta-\beta_{\mathrm{c}} D(A \cap B) \subseteq E_{c} D$ (A) $\cap E_{c-D} D(B)\left(\right.$ resp. $\left.\delta-\beta_{\mathrm{c}-} D(A) \cap \delta-\beta_{\mathrm{c}} D(B)\right)$.

VI. $\quad E_{c} D\left(E_{c-} D(A)\right)\left(\right.$ resp. $\delta-\beta_{\mathrm{c}} D\left(\delta-\beta_{\mathrm{c}} D(A)\right) A \subseteq E_{c-}$ $D(A)\left(\right.$ resp. $\left.\delta-\beta_{\mathrm{c}} D(A)\right)$.

VII. $\quad E_{c-} D\left(A \cup E_{c} D(A)\right) \subseteq A \cup E_{c} D(A)$.

VIII. $\quad \delta-\beta_{\mathrm{c}-} D\left(A \cup \delta-\beta_{\mathrm{c}} D(A)\right) \subseteq A \cup \delta-\beta_{\mathrm{c}} D(A)$.

Proposition4.16: For any subset $A$ of a space $X$, the following statements hold:

I. $E_{c-} D(A)\left(\right.$ resp. $\left.\delta-\beta_{\mathrm{c}} D(A)\right) \subseteq E_{c-} C l(A)($ resp. $\delta$ $\left.\beta_{\mathrm{c}-} C l(A)\right)$.

II. $\quad E_{c-} C l(A)\left(\right.$ resp. $\left.\delta-\beta_{\mathrm{c}-} C l(A)\right)=A \cup E_{c-} D(A)($ resp. $\left.A \cup \delta-\beta_{\mathrm{c}-} D(A)\right)$.

Theorem 4.17: Let $A$ be subset of a space $X$. Then, $A$ is $E_{c}$ (resp. $\delta$ - $\beta_{\mathrm{c}}$ )-closed set iff it contains all of its $E_{c}$ (resp. $\delta$ - $\boldsymbol{\beta}_{\mathrm{c}}$ )-Limit points.

Definition 4.18: Let $A$ be a subset of a topological space $(X, T)$. The $E_{c}$ (resp. $\delta$ - $\beta_{\mathrm{c}}$ )-Frontier of $A$, denoted by $E_{c-} F_{r}(A)\left(\operatorname{resp} . \delta-\beta_{\mathrm{c}} F_{r}(A)\right)$ is defined by:

$E_{c-} F_{r}(A)\left(\right.$ resp. $\left.\delta-\beta_{\mathrm{c}-} F_{r}(A)\right)=E_{c-} C l(A) \quad\left(\right.$ resp. $\delta-\beta_{\mathrm{c}-}$ $C l(A)) E_{c} \operatorname{Int}(A)\left(\right.$ resp. $\delta$ - $\left.\beta_{\mathrm{c}} \operatorname{Int}(A)\right)$.

Theorem 4.19: For any subset $A$ of a space $X$, the following properties hold:

I. $\quad E_{c-} C l(A)\left(\right.$ resp. $\delta$ - $\left.\beta_{\mathrm{c}-} C l(A)\right)=E_{c} \operatorname{Int}(A)($ resp. $\delta$ $\left.\beta_{\mathrm{c}} \operatorname{Int}(A)\right) \cup E_{c-} F_{r}(A)\left(\right.$ resp. $\left.\delta-\beta_{\mathrm{c}-} F_{r}(A)\right)$.

II. $\quad E_{c} \operatorname{Int}(A)\left(\operatorname{resp} . \delta-\beta_{\mathrm{c}} \operatorname{Int}(A)\right) \cap E_{c-} F_{r}(A)\left(\right.$ resp. $\delta-\beta_{\mathrm{c}-}$ $\left.F_{r}(A)\right)=\varphi$.

III. $\quad E_{c-} F_{r}(A)\left(\right.$ resp. $\left.\delta-\beta_{\mathrm{c}-} F_{r}(A)\right)=E_{c-} C l(A)\left(\right.$ resp. $\delta-\beta_{\mathrm{c}-}$ $C l(A)) \cap E_{c-} C l(X \backslash A)\left(\right.$ resp. $\left.\delta-\beta_{\mathrm{c}-} C l(X \backslash A)\right)$.

Corollary 4.20: For every subset $A$ of the space $X, E_{c}$ $F_{r}(A)\left(\right.$ resp. $\delta$ - $\left.\beta_{\mathrm{c}-} F_{r}(A)\right)$ is $E_{c}\left(\right.$ resp. $\delta$ - $\left.\beta_{\mathrm{c}}\right)$-closed set.

Now, we give new equivalent definitions for $E_{c}$ (resp. $\delta$ - $\beta_{\mathrm{c}}$ )-open sets and $E_{c}\left(\right.$ resp. $\delta$ - $\beta_{\mathrm{c}}$ )-closed sets by using the concept of $E_{c}$ (resp. $\delta$ - $\beta_{\mathrm{c}}$ )-Frontier in the following Theorem.

Theorem 4.21: The following properties hold for a subset $A$ of a topological space $(X, T)$ :

I. $\quad A$ is $E_{c}$ (resp. $\delta$ - $\beta_{\mathrm{c}}$ )-open $\Leftrightarrow A \cap E_{c-} F_{r}(A)$ (resp. $\left.\delta-\beta_{\mathrm{c}-} F_{r}(A)\right)=\varphi$. 
II. $\quad A$ is $E_{c}\left(\right.$ resp. $\delta$ - $\left.\beta_{c}\right)$-closed $\Leftrightarrow E_{c-} F_{r}(A)($ resp. $\delta$ $\left.\beta_{c-} F_{r}(A)\right) \subseteq A$.

III. $\quad A$ is both $E_{c}$ (resp. $\delta$ - $\beta_{c}$ )-open \& $E_{c}$ (resp. $\delta$ $\beta c)$-closed $\Leftrightarrow E_{c}$ Fr $(A)\left(\right.$ resp. $\left.\delta-\beta_{c-} F_{r}(A)\right)=\varphi$.

Proof: (i) - Assume that $A$ is $E_{c}$ (resp. $\delta$ - $\beta_{\mathrm{c}}$ )-open set, then from Theorem (4.6-part (iii)) we get $A=E_{c} \operatorname{Int}(A)$ (resp. $\delta$ - $\left.\beta_{\mathrm{c}} \operatorname{Int}(A)\right)$, also via Theorem (4.19), we have, $E_{c} \operatorname{Int}(A)\left(\operatorname{resp} . \delta-\beta_{\mathrm{c}} \operatorname{Int}(A)\right) \cap E_{c-} F_{r}(A)\left(\operatorname{resp} . \delta-\beta_{\mathrm{c}} F_{r}(A)\right)=$ $\varphi ; \Rightarrow A \cap E_{c-} F_{r}(A)\left(\right.$ resp. $\left.\delta-\beta_{\mathrm{c}-} F_{r}(A)\right)=\varphi$.

Conversely, suppose that $A \cap E_{c-} F_{r}(A)\left(\right.$ resp. $\left.\delta-\beta_{c} F_{r}(A)\right)$ $=\varphi$. Then,

$A \cap\left[E_{c-} C l(A) \quad\left(\right.\right.$ resp. $\left.\delta-\beta_{\mathrm{c}-} C l(A)\right) E_{c} \operatorname{Int}(A) \quad($ resp. $\delta$ $\left.\left.\beta_{\mathrm{c}} \operatorname{Int}(A)\right)\right]=A \cap\left[E_{c-} C l(A)\left(\operatorname{resp} . \quad \delta-\beta_{\mathrm{c}-} C l(A)\right)\right] \cap A \backslash[$ $\left.E_{c} \operatorname{Int}(A) \quad\left(\operatorname{resp} . \quad \delta-\beta_{\mathrm{c}} \operatorname{Int}(A)\right)\right]=A \backslash E_{c} \operatorname{Int}(A)($ resp. $\delta$ $\left.\beta_{\mathrm{c}} \operatorname{Int}(A)\right)=\varphi$. Hence $A=E_{c} \operatorname{Int}(A)\left(\operatorname{resp} . \delta-\beta_{\mathrm{c}} \operatorname{Int}(A)\right)$. Thus $A$ is $E_{c}$ (resp. $\delta$ - $\beta_{\mathrm{c}}$ )-open set.

(ii) - Suppose that $A$ is $E_{c}$ (resp. $\delta$ - $\beta_{\mathrm{c}}$ )-closed set, then from Theorem (4.9) $A=E_{c-} C l(A)\left(\right.$ resp. $\left.\delta-\beta_{\mathrm{c}-} C l(A)\right)$ and thus, $E_{c-} F_{r}(A)\left(\right.$ resp. $\left.\delta-\beta_{\mathrm{c}-} F_{r}(A)\right)=E_{c-} C l(A)\left(\right.$ resp. $\delta-\beta_{\mathrm{c}-}$ $C l(A)) E_{c} \operatorname{Int}(A)\left(\operatorname{resp} . \delta-\beta_{\mathrm{c}} \operatorname{Int}(A)\right)=A \backslash E_{c} \operatorname{Int}(A)($ resp. $\delta$ $\left.\beta_{\mathrm{c}} \operatorname{Int}(A)\right)$ and so, $E_{c-} F_{r}(A)\left(\operatorname{resp} . \delta-\beta_{c-} F_{r}(A)\right) \subseteq A$.

Conversely, let $E_{c-} F_{r}(A)\left(r e s p . \delta-\beta_{c}-F_{r}(A)\right) \subseteq A$, thus $E_{c-} C l(A) \quad\left(\right.$ resp. $\left.\quad \delta-\beta_{\mathrm{c}-} C l(A)\right)=E_{c} \operatorname{Int}(A) \quad($ resp. $\delta$ $\left.\beta_{\mathrm{c}} \operatorname{Int}(A)\right) \cup E_{c-} F_{r}(A)\left(\right.$ resp. $\left.\delta-\beta_{c}-F_{r}(A)\right) \subseteq E_{c} \operatorname{Int}(A)$ (resp. $\left.\delta-\beta_{\mathrm{c}} \operatorname{Int}(A)\right) \cup A=A$, as well we have by Theorem (4.11part (i)) $A \subseteq E_{c-} C l(A)$ (resp. $A \subseteq \delta$ - $\beta_{\mathrm{c}-} C l(A)$ ). Therefore, $E_{c-} C l(A)\left(\right.$ resp. $\left.\delta-\beta_{\mathrm{c}-} C l(A)\right)=A$ and via Theorem (4.9) we get $A$ is $E_{c}$ (resp. $\delta$ - $\beta_{c}$ )-closed set.

(iii) - suppose that $A$ is both $E_{c}\left(r e s p . \delta\right.$ - $\left.\beta_{c}\right)$-open and $E_{c}$ (resp. $\delta$ - $\beta_{c}$ )-closed. Therefore via Theorems' (4.6 and 4.9) we have, $E_{c} \operatorname{Int}(A)\left(\operatorname{resp} . \delta-\beta_{c} \operatorname{Int}(A)\right)=A=E_{c-} C l(A)$ (resp. $\left.\delta-\beta_{\mathrm{c}-} C l(A)\right)$ and by definition of $E_{c}\left(\right.$ resp. $\left.\delta-\beta_{\mathrm{c}}\right)$ Frontier of $A$, we get $E_{c} F_{r}(A)\left(\right.$ resp. $\left.\delta-\beta_{\mathrm{c}-} F_{r}(A)\right)=A \backslash A=$ $\varphi$. On the other hand, assume $E_{c-} F_{r}(A)$ (resp. $\delta-\beta_{c-}$ $\left.F_{r}(A)\right)=\varphi$, which means, $E_{c-} C l(A) \quad\left(\right.$ resp. $\delta-\beta_{\text {c- }}$ $C l(A)) E_{c} \operatorname{Int}(A)$ (resp. $\left.\delta-\beta_{\mathrm{c}} \operatorname{Int}(A)\right)=\varphi$, also in other words, $E_{c-} C l(A)\left(\right.$ resp. $\left.\delta-\beta_{\mathrm{c}-} C l(A)\right)=E_{c} \operatorname{Int}(A)($ resp. $\delta$ $\left.\beta_{\mathrm{c}} \operatorname{Int}(A)\right)$. But from Theorem (4.6-part (vi)) and Theorem (4.11-part (i)) We have, $E_{c} \operatorname{Int}(A)$ (resp. $\delta$ $\left.\beta_{\mathrm{c}} \operatorname{Int}(A)\right) \subseteq A$ and $A \subseteq E_{c-} C l(A)\left(\right.$ resp. $A \subseteq \delta$ - $\left.\beta_{\mathrm{c}-} C l(A)\right)$. Therefore, it is follow

$E_{c} \operatorname{Int}(A)\left(\operatorname{resp} . \delta-\beta_{\mathrm{c}} \operatorname{Int}(A)\right)=A=E_{c-} \operatorname{Cl}(A)\left(\operatorname{resp} . \delta-\beta_{\mathrm{c}-}\right.$ $C l(A))$ which means that $A$ is both $E_{c}$ (resp. $\delta$ - $\beta_{c}$ )-open and $E_{c}$ (resp. $\delta$ - $\beta_{c}$ )-closed set.

\section{CONCLUSION}

Generalized open and closed sets play a very a prominent role in general Topology and it applications. And many topologists worldwide are focusing their researches on these topics and this mounted to many important and useful results. Indeed a significant theme in general Topology, Real analysis and many other branches of mathematics concerns the variously modified forms of continuity, separation axioms etc by utilizing generalized open and closed sets. Some of the well-known notions and that expected it will have a wide applying in physics and Topology and their applications is the notion of e-open, $\delta$ - $\beta$-open, $E_{c}$-open, $\delta$ - $\beta_{c}$-open sets. The importance of general topological spaces rapidly increases in both the pure and applied directions it plays a significant role in data mining. One can observe the influence of general topological spaces also in computer science and digital topology [32], computational topology for geometric and molecular design, particle physics, high energy physics, quantum physics, and Superstring theory [32]. In this paper we introduced and studied new classes of generalized open sets called $E_{c}$-open and $\delta$ - $\beta_{c}$-open sets which may have very important applications in quantum particle physics, high energy physics and superstring theory [33]. Furthermore, the fuzzy topological version of the concepts and results introduced in this paper are very important. Since El-Naschie has shown that the notion of fuzzy topology has very important applications in quantum particle physics especially in related to both string theory and $\varepsilon^{\infty}$ theory $[34,35]$.

\section{ACKNOWLEDGEMENTS}

I would like to express my sincere gratitude to the referees for their valuable suggestions and comments which improved the paper and I am thankful to professor. Dr. E. Ekici (Turkey) for sending many of his papers as soon as I had requested and Dr. Yasir AlAni (University of Anbar-Iraq) for help.

\section{REFERENCES}

1. Hatir, E., \& Noiri, T. (2006). Decompositions of continuity and complete continuity. Acta Math Hungar. 113(4):281-287.

2. Ekici, E. (2009). On e*-Open Sets and (D, S)*Sets. Math. Moravica. 13(1):29-36.

3. Hariwan, Z. I. (2013). Bc-Open Sets in Topological Spaces. Advances in Pure Mathematics. 3:34-40.

4. Alias, B. K., \& Zanyar, A. A. (2010). Sc-open sets and Sc-continuity in topological spaces. Journal of Advanced Research in Pure Mathematics. 2(3):87101.

5. Zanyar, A. A. (2011). Pc-open sets and Pccontinuity in topological spaces. Journal of Advanced Research in Name. 3(1):1-12.

6. Ayman, Y. M. (2015). Continuity and Separation Axioms Based on Bc-Open Sets. M. Sci. Thesis, Islamic University of Gaza. Palestine.

7. Park, J. H. (2006). Strongly $\theta$-b-continuous functions. Acta Mathematica Hungarica. 110(4):347-359.

8. Alaa, M. F., \& Xiao, S. Y. (2015). New Type of Strongly Continuous Functions In topological Spaces via $\delta$-ß-open sets. European journal of pure and applied mathematics. 8(2):185-200.

9. Stone, M. H. (1937). Applications of the theory Boolean rings to general topology. Trans Amer Math Soc. 41:375-381.

10. Velicko, N. V. (1968). H-closed topological spaces. Amer Math Soc Transl. 2(78):103-118. 
11. Njastad, O. (1965). On some classes of nearly open sets. Pacific J Math. 15:961-970.

12. Noiri, T., \& Popa, V. (1993). Almost weakly continuous multi-functions. Demonstrati Math. 26:363-380.

13. Mashhour, A. S., Abd El-Monsef, M. E., \& ElDeeb, S. N. (1982). On pre-continuous and weak pre-continuous mappings. Proc Math Phy Soc Egypt. 53:47-53.

14. Abd El-Monsef, M. E., El-Deeb, S. N., \& Mahmoud, R. A. (1983). $\beta$-open sets and $\beta$ continuous mappings. Bull Fac Sci Assiut Univ. 12:77-90.

15. Andrijević, D. (1986). Semi-preopen sets. Mat Vesnik. 38:24-32.

16. Andrijević, D. (1996). On b-open sets. Mat Vesnik. 48:59-64.

17. El-Atik, A. A. (1997). A study of some types of mappings on topological spaces. M.Sci. Thesis, Tanta Uni. Egypt.

18. Raychaudhuri, S., \& Mukherjee, M. N. (1993). On $\delta$-almost continuity and $\delta$-Pre-open sets. Bull Inst Math Acad Sinica. 21:357-366.

19. Crossley, S. G., \& Hildebrand, S. K. (1971). Semiclosure. Texas J Sci. 22:99-112.

20. Mashhour, A. S., Hasanein, I. A., \& El-Deeb, S. N. (1983). On $\alpha$-continuous and $\alpha$-open Mappings. Acta Math Hungar. 41:213-218.

21. El-Deeb, S. N., Hasanein, I. A., Mashhour, A. S., \& Noiri, T. (1983). On p-regular spaces. Bull Math Soc Sci Math R Soc Roumanie. 72(75):311-315.

22. Ekici, E. (2008). On e-open sets, DP*-sets and DPE*-sets and decompositions of continuity. The Arabian J for Sci Eng. 33(2A):269-282.
23. Joseph, J., \& Kwach, M. (1980). On S-Closed Spaces. Proc Amer Math Soc. 80(2):341-348

24. Long, P., \& Herrington, L. (1982). The T $\theta$ Topology and Faintly Continuous Functions. Kyungpook Math J. 22 (1):7-14.

25. Dontchev, J. (1998). Survey on Pre-Open Sets, the Proceedings of the Yastsushiro Topological Conference. 1-18.

26. Willard, S. (1970). General Topology, University of Alberta, Addison Wesley Publishing Company, London.

27. Alexandroff, P. (1937). Diskrete Raume. Math Sbornik. 2:501-518

28. Hatir, E., \& Noiri, T. (2009). On $\delta$-ß-continuous functions. Chaos, Solitons and Fractals. 42:205-211.

29. Ekici, E. (2007). Some generalizations of almost contra-super-continuity. Filomat. 21(2):31-44.

30. Ekici, E. (2004). ( $\delta$-pre, s)-Continuous Functions. Bull. Malays. Math Sci Soc. 27(2):237-251.

31. Sharma, J. N. (1977). Topology. Krishna Parkashan Mandir. (2).

32. El-Naschie, M. S. (2005). The two slit experiment as the foundation of E-infinity of high energy physics. Chaos, Solitons \& Fractals. 25:509-514.

33. El-Naschie, M. S. (2006). Topics in the mathematical physics of E-infinity theory. Chaos, Solitons \& Fractals. 30(3): 656-663.

34. El-Naschie, M. S. (2000). On the unification of heterotic strings, M theory and theory. Chaos, Solitons \& Fractals. 11:2397-2408.

35. El-Naschie, M. S. (2004). A review of E-infinity theory and the mass spectrum of high energy particle physics. Chaos, Solitons \& Fractals. 19:209-36. 